\title{
The star formation rate density and dust attenuation evolution over 12 Gyr with the VVDS surveys ${ }^{\star, \star \star}$
}

\author{
O. Cucciati ${ }^{1,2}$, L. Tresse ${ }^{1}$, O. Ilbert ${ }^{1}$, O. Le Fèvre ${ }^{1}$, B. Garilli ${ }^{1,3}$, V. Le Brun ${ }^{1}$, P. Cassata ${ }^{4}$, P. Franzetti ${ }^{3}$, D. Maccagni ${ }^{1,3}$, \\ M. Scodeggio ${ }^{3}$, E. Zucca ${ }^{5}$, G. Zamorani ${ }^{5}$, S. Bardelli ${ }^{5}$, M. Bolzonella ${ }^{5}$, R. M. Bielby ${ }^{6,7}$, H. J. McCracken ${ }^{7}$, \\ A. Zanichelli ${ }^{8}$, and D. Vergani ${ }^{5}$ \\ (Affiliations can be found after the references)
}

Received 3 September 2011 / Accepted 31 December 2011

\begin{abstract}
Aims. We investigate the global galaxy evolution over $\sim 12$ Gyr $(0.05 \leq z \leq 4.5)$, from the far ultraviolet (FUV) luminosity function (LF), luminosity density (LD), and star formation rate density (SFRD), using the VIMOS-VLT Deep Survey (VVDS), a single deep galaxy redshift survey with a well controlled selection function.

Methods. We combine the VVDS Deep $\left(17.5 \leq I_{A B} \leq 24.0\right)$ and Ultra-Deep $\left(23.00 \leq i_{A B}^{\prime} \leq 24.75\right)$ redshift surveys, totalizing $\sim 11000$ galaxies, to estimate the rest-frame FUV LF and LD, using a wide wavelength range of deep photometry $(337<\lambda<2310 \mathrm{~nm})$. We extract the dust attenuation of the FUV radiation, embedded in the well-constrained spectral energy distributions. We then derive the dust-corrected SFRD.

Results. We find a constant and flat faint-end slope $\alpha$ in the FUV LF at $z<1.7$. At $z>1.7$, we set $\alpha$ steepening with $(1+z)$. The absolute magnitude $M_{\mathrm{FUV}}^{*}$ steadily brightens in the entire range $0<z<4.5$, and at $z>2$ it is on average brighter than in the literature, while $\phi^{*}$ is on average smaller. The evolution of our total LD shows a peak at $z \simeq 2$, clearly present also when considering all sources of uncertainty. The SFRD history peaks as well at $z \simeq 2$. It first steadily rises by a factor of $\sim 6$ during $2 \mathrm{Gyr}$ (from $z=4.5$ to $z=2$ ), and then decreases by a factor of $\sim 12$ during $10 \mathrm{Gyr}$ down to $z=0.05$. This peak is mainly produced by a similar peak within the population of galaxies with $-21.5 \leq M_{\mathrm{Fuv}} \leq-19.5$. As times goes by, the total SFRD is dominated by fainter and fainter galaxies. The mean dust attenuation of the global galaxy population rises fast by 1 mag during $2 \mathrm{Gyr}$ from $z \simeq 4.5$ to $z \sim 2$, reaches slowly its maximum at $z \simeq 1$ ( $A_{\mathrm{FuV}} \simeq 2.2 \mathrm{mag}$ ), and then decreases by $1.1 \mathrm{mag}$ during $7 \mathrm{Gyr}$ down to $z \simeq 0$. Conclusions. We have derived the cosmic SFRD history and the total dust amount in galaxies over a continuous period of $\sim 12 \mathrm{Gyr}$, using a single homogeneous spectroscopic redshift sample. The presence of a clear peak at $z \simeq 2$ and a fast rise at $z>2$ of the SFRD is compelling for models of galaxy formation. This peak is mainly produced by bright galaxies $\left(L \gtrsim L_{z=2}^{*}\right)$, requiring that significant gas reservoirs still exist at this epoch and are probably replenished by cold accretion and wet mergers, while feedback or quenching processes are not yet strong enough to lower the SF. The dust attenuation maximum is reached $\sim 2$ Gyr after the SFRD peak, implying a contribution from the intermediate-mass stars to the dust production at $z<2$.
\end{abstract}

Key words. cosmology: observations - galaxies: evolution - galaxies: high-redshift - galaxies: luminosity function, mass function galaxies: star formation

\section{Introduction}

A robust determination of the star formation rate history (SFRH) is a crucial element to understand galaxy evolution. As time goes by, the reservoirs of pristine gas are transformed into first generation of stars. During galaxy evolution, feedback processes, gas accretion, mergers, and environment are expected to affect star formation, and to contribute as well to galaxy mass assembly.

* Based on observations collected at the European Organisation for Astronomical Research in the Southern Hemisphere, Chile under programs 072.A-0586 (GTO), 073.A-0647 (GTO) and 177.A-0837 (LP) at the Very Large Telescope, Paranal, and based on observations obtained with MegaPrime/MegaCam, a joint project of CFHT and CEA/DAPNIA, at the Canada-France-Hawaii Telescope (CFHT) which is operated by the National Research Council (NRC) of Canada, the Institut National des Science de l'Univers of the Centre National de la Recherche Scientifique (CNRS) of France, and the University of Hawaii. This work is based in part on data products produced at TERAPIX and the Canadian Astronomy Data Centre as part of the Canada-France-Hawaii Telescope Legacy Survey, a collaborative project of NRC and CNRS.

$\star \star$ Appendices are available in electronic form at

http://www. aanda.org
The SFH therefore contains an imprint of the collective outcome of all processes which shape galaxies along time. The study of the SFH has been pioneered by the CFRS (Lilly et al. 1996), on the evolution of the luminosity density since $z \sim 1$, and by Madau et al. (1996), on the SFR using the CFRS and $z \sim 3-4$ samples identified with HST. Since then, extensive new SFRD measurements have been compiled up to $z \simeq 6$ (see, for example, Hopkins \& Beacom 2006).

The star formation rate density (SFRD) is usually derived from a mean luminosity density, defined as $\mathrm{LD}=\int_{0}^{\infty} \phi(L) L \mathrm{~d} L$, with $\phi(L)$ being the luminosity function (LF) and $L$ a luminosity related to the SFR. At first sight, the SFRD is a simple and powerful tool to investigate the cosmic star formation history. The various data from different samples have persistently shown a broad picture consistent within factors of $\sim 3$, out to highredshifts $(z \simeq 6)$, showing a rise out to $z \simeq 1$ and a decline from $z \simeq 3$, with an unclear evolution within $1 \lesssim z \lesssim 2.5$ because this redshift desert has remained difficult to probe. From the present day to $z \simeq 1$, a steady rise of the SFRD by one order of magnitude is firmly corroborated using various calibrators of SFR like far ultraviolet (FUV), far infrared (FIR), H $\alpha$, radio - but the scatter amongst different measurements remains large. Because 
of the number of uncertainties that remain along the chain of transformations to be applied to galaxy counts and luminosities to be converted into star formation rates, the exact shape of the SFRD evolution still remains to be established. The selection function of each galaxy sample, including the imaging surveys depth and image quality or the redshift completeness, requires a number of corrections to compute volume densities, and the complete shape of the luminosity function remains speculative, particularly at the faint end. Moreover, the transformation of luminosities to SFR depends on assumptions on the conversion factors and on the amount of dust attenuation, still open issues.

To be able to derive a coherent galaxy evolution model of galaxy evolution, it is necessary to trace the SFRD evolution with the same reference indicator throughout cosmic time, and within a deep galaxy redshift survey with a simple, well defined and controlled selection function.

The selection of spectroscopic targets may have a strong impact on the type of galaxies studied and hence on the relevance of this sample to the full galaxy population. A tight control of the selection function is necessary to avoid the propagation of biases to some types of galaxies or redshift ranges. Several methods are used to pre-select galaxies, either using colour criteria tailored to different redshift ranges and populations (e.g. LBG, $\mathrm{BzK}$, radio, far-IR,...), or pure magnitude selection. A follow-up spectroscopic survey with high success rate has then the advantage to provide a sample of galaxies with known redshift and controlled uncertainties, with stars and broad-line active galaxy nuclei clearly identified as pre-selection techniques are largely unable to fully discard them.

Using rest-frame ultraviolet galaxy luminosities has become a common approach at high redshift, as it is applicable up to the highest redshifts studied so far $(z \simeq 7$, e.g. Bouwens et al. 2009). The non-ionising ultraviolet light $(912-3000 \AA)$ is emitted by relatively massive $\left(\geq 3 M_{\odot}\right)$, short-lived $\left(<3 \times 10^{8} \mathrm{yr}\right)$ stars and it traces the SFR averaged over the last $\sim 10^{8}$ yr once it is corrected for dust attenuation (Kennicutt 1998). This SFR estimator assumes a constant SFR over longer times than the very massive stellar population ( $\gg 15 M_{\odot}$ ) contributing to the $\mathrm{H} \alpha$ emission. As opposed to the $\mathrm{H} \alpha$ instantaneous SFR estimator, it does not disentangle whether the radiation is linked to the creation of new stars $\left(<10^{7} \mathrm{yr}\right)$ or to more evolved stars $\left(<10^{8} \mathrm{yr}\right)$, and thus it is sensitive to ageing of star formation regions (Calzetti 2008). An ideal measurement of the instantaneous SFR, not affected by uncertainties on dust, would be based on the simultaneous use of $\mathrm{H} \alpha$ and infrared emissivities, since the absorbed ionising flux heats the dust which re-emits in the infrared (Calzetti 2008). The difficulty is that it has not yet been possible to assemble large, deep and statistically complete samples for both estimators.

Here we aim to derive the SFRD evolution since $z \sim 4.5$ using the rest-frame UV luminosity density from the VIMOS VLT Deep Survey (VVDS) samples “Deep" and "Ultra-Deep", totalizing $\sim 11000$ galaxies with spectroscopic redshifts. We take advantage of being able to compute rest-frame ultraviolet emissivities over a large and deep area of sky, which enables to trace its evolution in a consistent way since $z \simeq 4.5$.

This paper is organised as follows. Section 2 gives a summary of our VVDS data sets. Sections 3 and 4 describe the ultraviolet luminosity functions (LF) and densities (LD) from $z=0.05$ to $z=4.5$ as derived from these data. Section 5 presents our results for the evolution of the dust attenuation and the dust-corrected SFRD in the past $\sim 12$ Gyr. Section 6 summarises and discusses our results. Technical details are given in the Appendices. Throughout this paper, we use the AB flux normalisation, and we adopt the concordance cosmology $\left(\Omega_{\mathrm{m}}\right.$, $\left.\Omega_{\Lambda}, h\right)=(0.3,0.7,0.7)$, with which the age of the Universe is 5.7, 3.2, 1.3 Gyr at $z=1,2,4.5$.

\section{Data}

\subsection{The VVDS Deep and Ultra-Deep spectroscopic surveys}

The VIMOS-VLT Deep Survey (VVDS) is a spectroscopic investigation of distant sources, carried out with the highmultiplex, wide-field (224 $\left.\operatorname{arcmin}^{2}\right)$ VIsible Multi-Object Spectrograph (VIMOS, Le Fèvre et al. 2003) mounted at the Nasmyth focus of MELIPAL, the third of the four $8.2 \mathrm{~m} \mathrm{ESO-}$ VLT Unit Telescopes in Paranal, Chile. The VVDS is composed of three $I$-selected surveys totalizing about 47000 spectra of galaxies, quasars, and stars; (1) a Wide survey (17.5 $\leq I_{A B} \leq$ 22.5, Garilli et al. 2008), (2) a Deep survey (17.5 $\leq I_{A B} \leq 24.0$, Le Fèvre et al. 2004b, 2005b), and (3) an Ultra-Deep survey $\left(23.00 \leq i_{A B}^{\prime} \leq 24.75\right.$, Le Fèvre et al., in prep.). It spans a wide redshift range; from $z>0$ to $z \simeq 5$ for targeted sources, and up to $z \simeq 6.6$ for serendipitous Ly $\alpha$ emitters (Cassata et al. 2011). The VVDS surveys are purely flux limited, and are free from any colour pre-selection or galaxy-star separation when preparing the target catalogues. In the Deep survey, the projection of galaxy size on the $x$-axis of the image has been used to maximise the number of targets in each VIMOS pointing (see Ilbert et al. 2005 , for further details). We refer the reader to McCracken et al. (2003) for a complete discussion on photometric completeness as a function of $I_{A B}$ magnitude and surface brightness. The conclusion of their analysis is that the VIMOS Deep Imaging Survey "is essentially free of selection effects until at least $I_{A B}=25$ ". Namely, considering the flux limit of the Ultra-Deep survey, $I_{A B} \leq 24.75$, our photometric catalogue is $90 \%$ and $70 \%$ complete in surface brightness down to 24.5 and $25 \mathrm{mag} / \operatorname{arcsec}^{2}$, respectively.

In this study, we use both the Deep and Ultra-Deep surveys obtained in the VVDS-0226-04 field. The Deep spectra were collected with integration times of $16000 \mathrm{~s}$ using the LR-Red grism $(R=210,5500<\lambda<9500 \AA)$, over $2200 \operatorname{arcmin}^{2}$ of sky area. They include the 9842 spectra described in Le Fèvre et al. (2005b), plus 2826 spectra acquired later with the same set-up (Le Fèvre et al., in prep.). With the Ultra-Deep survey we obtained repeated observations of several $z \geq 1.4$ targets from the Deep survey with assigned VVDS quality flags of 0,1 , or 2 (i.e., with the lowest confidence level in the spectroscopic identification) in order to assess their real redshift distribution as detailed in Le Fèvre et al. (in prep.). The Ultra-Deep spectra were collected using both the LR-Red grism and the LR-Blue grism $(3700<\lambda<6700 \AA, R=180)$ with integration times of $65000 \mathrm{~s}$ in each set-up. They consists of 1200 new targets acquired over $576 \operatorname{arcmin}^{2}$ of sky area within the $2200 \operatorname{arcmin}^{2}$ sampled by the Deep survey. Our present work is based on 10141 and 622 galaxy spectra at $0.05<z \leq 4.50$ from the Deep and Ultra-Deep surveys, respectively. Those spectra have a spectroscopic identification at a confidence level higher than $\sim 50 \%, 60 \%, 81 \%, 97 \%$ and $99 \%$ (corresponding to the VVDS quality flags $1,9,2,3$ and 4).

We do not have a measured redshift for every source to a given apparent magnitude limit in the observed field of view; the averaged target sampling rates are $\sim 24 \%$ and $\sim 4 \%$ for the Deep $\left(17.5 \leq I_{A B} \leq 24.0\right)$ and Ultra-Deep (23.00 $\left.\leq i_{A B}^{\prime} \leq 24.75\right)$ surveys, respectively. For each survey, we accurately estimated the selection function accounting for the facts that (i) a fraction of sources of the parent photometric catalogue was targeted for 
spectroscopic observations; (ii) a fraction of the targeted sources yields reliable redshifts. The computation of the selection functions practically translates into weights applied to each galaxy when computing the luminosity functions. In addition, in the Deep survey, we used the results from our repeated observations, to obtain a $100 \%$ confidence level in the redshift measurement of lower quality spectra. The weighting schemes for the Deep and Ultra-Deep surveys are independent, and we refer the reader to Appendices A.1 and A.2 for a detailed description.

\subsection{The absolute magnitudes}

For the astrometric and photometric catalogues, we take the following broadband imaging surveys over the VVDS-0226-04 field acquired at the Canada-France-Hawaii Telescope (CFHT) using wide-field mosaic cameras: the BVI VVDS imaging survey (McCracken et al. 2003; Le Fèvre et al. 2004a) with the CFHT-12K camera, the $u^{*} g^{\prime} r^{\prime} i^{\prime} z^{\prime}$ CFHT-Legacy Survey (CFHTLS-D1/W1 field, T0005 release) with the MEGACAM camera and the $J H K_{\mathrm{s}}$ WIRDS survey (Bielby et al. 2011) with the WIRCAM camera.

To derive intrinsic luminosities, we use our Algorithm for Luminosity Function (ALF, Ilbert et al. 2005), that integrates routines of the code Le Phare ${ }^{1}$. Absolute magnitude measurements are optimised accounting for the full information given by the above multi-band photometric data, in a way which minimises the dependency on the templates chosen to fit the observed colours (see A.1 in Ilbert et al. 2005). We choose the template library from Bruzual \& Charlot (2003) modulated by the attenuation of the intrinsic stellar continuum, $A(\lambda)=$ $k(\lambda) E(B-V)$. We consider a grid for the intrinsic colour excess of the continuum light $(E(B-V)=0,0.1,0.2,0.3,0.4$ and $0.5 \mathrm{mag})$, and an empirical attenuation curve, $k(\lambda)$, of the form defined by Calzetti et al. (2000). We verified that the absolute magnitudes are indeed mostly independent from the chosen template set, especially for those derived in the rest-frame FUV, NUV, $U$ and $B$ photometric bands. For this purpose, we computed them again using different template sets, like PEGASE. 2 templates (Fioc \& Rocca-Volmerange 1997) or a mixed set with Bruzual \& Charlot (2003) and Polletta et al. (2007) templates, as used in Ilbert et al. (2009). This very weak dependency of the derived absolute magnitudes on the adopted templates is mainly due to the fact that we use $i)$ a wide $(337<\lambda<2310 \mathrm{~nm})$ wavelength range with the observed $u^{*} B g^{\prime} V r^{\prime} i^{\prime} I z^{\prime} J H K_{\mathrm{s}}$ photometric broadbands to obtain a robust template fit, and $i i)$ the $u^{*}$-CFHTLS broadband filter (our bluest photometric information, $337<\lambda<411 \mathrm{~nm}$ ) that begins to sample the non-ionising ultraviolet continuum $(91.2-300 \mathrm{~nm})$ at $z>0.1$. The $u^{*}$ photometric information is deep enough to be complete down to our VVDS spectroscopic limiting magnitudes, based on the primary selection from the $I$-band CFHT-12K images $(725<\lambda<930 \mathrm{~nm})$. This is the case at least up to $z \simeq 3.5$ when the Lyman-break feature is shifted towards our redder photometric bands, i.e., $B g^{\prime} V r^{\prime} i{ }^{\prime} I$. The observed $u^{*}-381$ corresponds to the rest-frame NUV-250 at $z \simeq 0.4$ and to the rest-frame FUV-150 at $z \simeq 1.5$. It spans the NUV-250 band $(184<\lambda<280 \mathrm{~nm})$ from $z=0.2$ to $z=1.2$ and the FUV-150 band $(135<\lambda<175 \mathrm{~nm})$ from $z=0.9$ to $z=2$. At $z>2$, the rest-frame FUV-150 and NUV-250 luminosities are covered by observations with filters redder than $u^{*}$ (up to the $J$ band at $z=4$ for the NUV-250). Our rest-frame FUV absolute magnitudes are based on template extrapolation only for $z<1$,

\footnotetext{
1 http://www . cfht . hawaii . edu/ arnouts/LEPHARE/lephare. html
}

but still we verified that our FUV-based results agree with our NUV-based ones. As an example of these consistency tests, in Appendix B we compare the rest-frame NUV and FUV luminosity functions.

\section{The ultraviolet Deep and Ultra-Deep luminosity functions}

In this work, we have primarily estimated the rest-frame FUV luminosity functions and densities to derive the SFRD history. The recent SFR is traced by the intrinsic non-ionising ultraviolet stellar continuum (91.2-300 nm) of galaxies (see Sect. 1). Within this UV range, the far UV radiation (FUV-150) is a better SFR indicator than the near UV radiation (NUV-250), because the NUV is contaminated by evolved stars, while the FUV is dominated by the radiation from new, massive, short-lived stars (see, e.g., Madau et al. 1998).

\subsection{The method}

The galaxy luminosity function (LF) usually follows a Schechter (1976) function characterised by a break luminosity, $L^{*}$, a faintend slope, $\alpha$, and a normalisation density parameter, $\phi^{*}$. The LF is a fundamental measurement of the statistical properties of the galaxy population; it is the distribution of the galaxy comoving number density as a function of their intrinsic luminosity at a given epoch. Despite its simple definition, its estimation requires careful analyses of the survey strategy, the selection criteria, and the completeness. We derive it using our code ALF that includes the non-parametric $1 / V_{\max }, \mathrm{SWML}$, and $C^{+}$and the parametric STY luminosity function estimators (see Appendices in Ilbert et al. 2005, and references therein). Each estimator presents advantages and drawbacks, and each one is affected differently by different visibility limits for the various galaxy types detected in deep flux-limited surveys. In a given observed band, galaxies are not equally visible to the same absolute magnitude limit mainly due to the spectral type dependency of the $K$ corrections (i.e., different spectral energy distribution - SED - for different spectral type galaxies). Within a given redshift range, the use of several non-parametric LF estimators allows us to empirically determine the absolute magnitude range in which galaxies are equally visible within our spectroscopic surveys. The luminosity limit down to which all galaxy populations are visible is called LF bias. As discussed in Ilbert et al. (2004), $1 / V_{\max }$ and $\mathrm{C}^{+}$methods are affected by this bias in a different way than the SWML and STY methods. If $1 / V_{\max }$ or $C^{+}$at a given luminosity starts giving different results from the SWML or STY, it means that a bias in the global LF is present. The bias is significant if the two estimators differ of more than the statistical uncertainties (Poisson errors, in our case). In each redshift bin explored, we set the LF bias at the brightest absolute magnitude where $1 / V_{\max }$ or $C^{+}$are different by more than $1-\sigma$ from SWML or STY. Our LF parameters are estimated with data brighter than the LF bias limit, to derive an unbiased LF faint-end slope of the global population. This is particularly important when combining surveys of different depth. We use the Schechter (1976) functional form for the STY estimate to calculate the Schechter parameters, because the results are more robust than those with a simple fit with a Schechter function of the non-parametric LF data points. The resulting faint-end slope, $\alpha$, is independent on the luminosity binning, and since the LF parameters are highly correlated to each other, we can account for the allowed range for each 


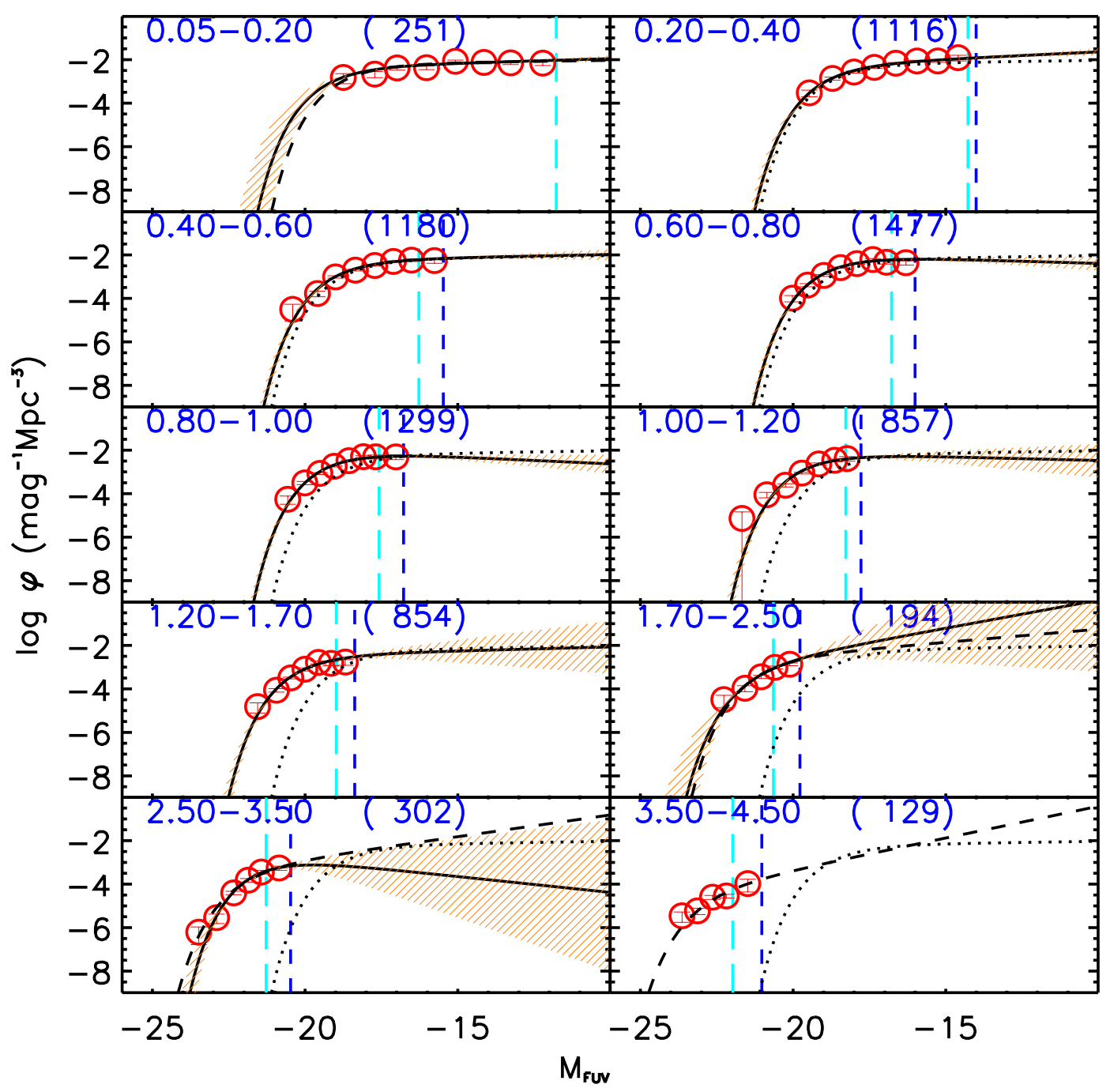

Fig. 1. Rest-frame FUV-band luminosity functions in ten redshift bins from $z=0.05$ to $z=4.50$ of the merged Deep+Ultra-Deep VVDS sample. Redshift ranges are indicated in each panel. Red circles represent the $1 / V_{\max }$ data points with Poisson error bars, and the number of galaxies is given in parenthesis. $1 / V_{\max }$ points are plotted up to the LF bias limit (see Sect. 3.1) of the merged sample, represented with a vertical blue short-dashed line. This line corresponds also to the LF bias limit of the Ultra-Deep sample. The vertical cyan long-dashed line corresponds to the LF bias limit of the Deep survey. There is no Ultra-Deep data at $0.05<z \leq 0.2$, so the only bias limit is the one of the Deep sample. The black solid curve and its associated orange shaded area is the STY LF estimate assuming free Schechter parameters and its associated error. At $3.5<z \leq 4.5$, the STY LF fit does not converge using all free parameters. In the first redshift bin, the dashed curve is the STY LF estimate when setting $M_{\mathrm{FUV}}^{*}=-18.12$ (see text), and for reference it is reported as a dotted curve in the other panels. In the $1.7<z \leq 2.5,2.5<z \leq 3.5$, $3.5<z \leq 4.5$ panels, the dashed curve is the STY LF estimate when setting a faint-end slope that evolves with increasing redshift (i.e., $\alpha=-1.3$, $-1.5,-1.73$, respectively). The Schechter parameters of all the curves shown in this plot are listed in Table B.1, while Table 1 summarises those that we retained for our study.

Schechter parameter as derived by the likelihood, in addition to the Poisson uncertainties typically quoted.

\subsection{The UV luminosity functions}

We compute the rest-frame FUV LFs using a unique merged catalogue which includes both the Deep and Ultra-Deep surveys, for a total covered magnitude range of $17.5 \leq I_{A B} \leq 24.75$. This way, we exploit both the large magnitude range covered by the Deep survey and the depth reached by the Ultra-Deep survey. This leads us to a robust determination of the LF shape and normalisation.

When using flux-limited surveys with various apparent luminosity depths, a coherent weighting scheme must take into account the possible overlap of the flux ranges covered by the different surveys. We detail the weights to be applied in this case in Appendix A.3, and we verify their robustness in Appendix A.4. Figure 1 shows the rest-frame FUV-band LFs $\left(1 / V_{\max }\right.$ and STY) obtained with the merged catalogue with $17.50 \leq I_{A B} \leq 24.75$, in ten independent redshift bins from $z=0.05$ to $z=4.5$. The non-parametric $1 / V_{\max }$ data points are plotted up to the LF bias limit, that is where those from the SWML and $\mathrm{C}^{+}$methods are in agreement (the latter estimates are not shown throughout the paper for clarity in the figures). At $z<0.2$, due to the size of our survey fields, rare bright nearby galaxies are not observed and the bright-end of the LF cannot be constrained as shown by the large error (shaded area in Fig. 1) associated to the STY estimate. For this reason, in the first redshift bin we set $M^{*}$ to the local FUV value, $M_{\mathrm{FUV}}^{*}=-18.12$ (Wyder et al. 2005). Conversely, at $z>1.7$, the faint-end of the LF starts to be loosely constrained. Recent results in the 
Table 1. LF Schechter parameters, LF absolute magnitude bias limits, LDs, SFRDs and dust attenuation derived from our rest-frame FUV-150 LF estimates in 10 redshift bins from $z=0.05$ to $z=4.5$ assuming $\left(\Omega_{\mathrm{m}}, \Omega_{\Lambda}, h\right)=(0.3,0.7,0.7)$.

\begin{tabular}{lcccccccc}
\hline \hline$\Delta z^{a}$ & $\begin{array}{c}M_{\mathrm{FUV}}^{*}{ }^{b} \\
\mathrm{AB} \mathrm{mag}\end{array}$ & $\alpha^{b}$ & $\begin{array}{c}\phi^{* b} \\
/ 10^{3} \mathrm{Mpc}^{3}\end{array}$ & $\begin{array}{c}M_{\mathrm{Bias}}^{\mathrm{D}}, M_{\mathrm{Bias}}^{\mathrm{UD} c} \\
\mathrm{AB} \text { mag }\end{array}$ & $\begin{array}{c}\lg \left(\mathrm{LD}_{\mathrm{uc}}\right)^{d} \\
\mathrm{~W} / \mathrm{Hz} / \mathrm{Mpc}^{3}\end{array}$ & $\begin{array}{c}\lg \left(\mathrm{SFRD}_{\mathrm{uc}}\right)^{e} \\
M_{\odot} / \mathrm{yr} / \mathrm{Mpc}^{3}\end{array}$ & $\begin{array}{c}A_{\mathrm{FUV}^{f}} \\
\mathrm{mag}\end{array}$ & $\begin{array}{c}\lg \left(\mathrm{SFRD}_{\mathrm{c}}\right)^{g} \\
M_{\odot} / \mathrm{yr} / \mathrm{Mpc}^{3}\end{array}$ \\
\hline $0.05-0.2$ & -18.12 & $-1.05_{-0.04}^{+0.04}$ & $7.00_{-0.44}^{+0.44}$ & $-11.8, \ldots$ & $18.76_{-0.18}^{+0.18}$ & $-2.09_{-0.18}^{+0.18}$ & 1.11 & $-1.65_{-0.18}^{+0.18}$ \\
$0.2-0.4$ & $-18.3_{-0.2}^{+0.1}$ & $-1.17_{-0.05}^{+0.05}$ & $6.91_{-0.95}^{+1.02}$ & $-14.3,-14.0$ & $18.87_{-0.12}^{+0.12}$ & $-1.98_{-0.12}^{+0.12}$ & 1.35 & $-1.44_{-0.12}^{+0.12}$ \\
$0.4-0.6$ & $-18.4_{-0.1}^{+0.1}$ & $-1.07_{-0.06}^{+0.07}$ & $6.60_{-0.86}^{+0.91}$ & $-16.3,-15.5$ & $18.85_{-0.10}^{+0.10}$ & $-2.00_{-0.10}^{+0.10}$ & 1.64 & $-1.34_{-0.10}^{+0.10}$ \\
$0.6-0.8$ & $-18.3_{-0.1}^{+0.1}$ & $-0.90_{-0.08}^{+0.08}$ & $9.53_{-0.99}^{+0.99}$ & $-16.8,-16.0$ & $18.93_{-0.09}^{+0.09}$ & $-1.92_{-0.09}^{+0.09}$ & 1.92 & $-1.15_{-0.09}^{+0.09}$ \\
$0.8-1.0$ & $-18.7_{-0.1}^{+0.1}$ & $-0.85_{-0.10}^{+0.10}$ & $9.01_{-0.96}^{+0.94}$ & $-17.6,-16.8$ & $19.04_{-0.08}^{+0.09}$ & $-1.79_{-0.08}^{+0.09}$ & 2.22 & $-0.90_{-0.08}^{+0.09}$ \\
$1.0-1.2$ & $-19.0_{-0.2}^{+0.2}$ & $-0.91_{-0.16}^{+0.16}$ & $7.43_{-1.15}^{+1.08}$ & $-18.3,-17.8$ & $19.12_{-0.09}^{+0.09}$ & $-1.74_{-0.09}^{+0.09}$ & 2.21 & $-0.85_{-0.09}^{+0.09}$ \\
$1.2-1.7$ & $-19.6_{-0.2}^{+0.2}$ & $-1.09_{-0.23}^{+0.23}$ & $4.10_{-0.87}^{+0.77}$ & $-19.0,-18.4$ & $19.13_{-0.08}^{+0.15}$ & $-1.72_{-0.08}^{+0.15}$ & 2.17 & $-0.85_{-0.08}^{+0.15}$ \\
$1.7-2.5$ & $-20.4_{-0.1}^{+0.1}$ & -1.30 & $3.37_{-0.24}^{+0.24}$ & $-20.6,-19.8$ & $19.46_{-0.09}^{+0.49}$ & $-1.40_{-0.09}^{+0.49}$ & 1.94 & $-0.62_{-0.09}^{+0.49}$ \\
$2.5-3.5$ & $-21.4_{-0.1}^{+0.1}$ & -1.50 & $0.86_{-0.05}^{+0.05}$ & $-21.3,-20.5$ & $19.40_{-0.15}^{+0.26}$ & $-1.45_{-0.15}^{+0.26}$ & 1.47 & $-0.86_{-0.15}^{+0.26}$ \\
$3.5-4.5$ & $-22.2_{-0.2}^{+0.2}$ & -1.73 & $0.11_{-0.01}^{+0.01}$ & $-22.0,-21.2$ & $19.10_{-0.32}^{+0.22}$ & $-1.76_{-0.32}^{+0.22}$ & 0.97 & $-1.37_{-0.32}^{+0.22}$ \\
\hline
\end{tabular}

Notes. Values derived for the merged VVDS Deep+Ultra-Deep sample at $17.50 \leq I_{A B} \leq 24.75$. There is no Ultra-Deep data at $z<0.2$. $^{(a)}$ Redshift bins. ${ }^{(b)}$ Schechter parameters $\left(M^{*}, \alpha\right.$ and $\left.\phi^{*}\right)$ of our VVDS rest-frame FUV-150 luminosity function that we use throughout this work. ${ }^{(c)}$ Absolute magnitude limit in FUV down to which the survey is complete is terms of galaxy types (see Sect. 3.1), for the Deep and the Ultra-Deep surveys, respectively. ${ }^{(d)}$ Rest-frame FUV luminosity density uncorrected for dust. The quoted uncertainty includes errors on the LD induced by the STY LF fit, the Poisson noise, the cosmic variance, and the weighting scheme. When $\alpha$ is set at $z>1.7$, the STY errors include also the percentage uncertainty accounting for the two extreme values of $\alpha(-1.1,-1.73)$ with respect to those quoted in the third column (see text). The relative contribution of each source of error is detailed in Table 2. ${ }^{(e)}$ Star formation rate density uncorrected for dust, derived from the restframe FUV luminosity density. For the errors see note $(d) .{ }^{(f)}$ Average dust attenuation in FUV. ${ }^{(g)}$ Star formation rate density corrected for the dust attenuation $A_{\mathrm{FUV}}$. For the errors see note (d).

literature (see Table B.2) seem to indicate a steep faint-end slope of the rest-frame FUV-band LF at $z \gtrsim 2$. We therefore opt for a slope set to evolve with increasing redshift, using the parameterisation, $\alpha(z)=A(1+z)+B$ (see, e.g., Ryan et al. 2007). To derive $A$ and $B$, we use our highest redshift estimate of $\alpha(\alpha=-1.1$ at $z \sim 1.45$, see Table B.1) and $\alpha=-1.73$ at $z=3.8$ taken from Bouwens et al. (2007). In this way, we obtain $\alpha=-1.3,-1.5$, and -1.73 at $1.7<z \leq 2.5,2.5<z \leq 3.5$, and $3.5<z \leq 4.5$, respectively. In addition, in all the redshift bins at $1.7<z \leq 4.5$ we compute $\phi^{*}$ and $M^{*}$ using also extreme non-evolving $\alpha$ cases: $\alpha=-1.1$ as estimated at $1.2<z \leq 1.7$, and $\alpha=-1.73$, as estimated at $z \sim 3.8$ by Bouwens et al. (2007). Table B.1 summarises the Schechter parameters for all above cases.

From now on, we consider our final best Schechter parameters $\left(\phi^{*}, M^{*}, \alpha\right)$ of the rest-frame FUV LFs as those estimated with the STY method with $M^{*}$ set at the local value at $0.05<z \leq 0.2$, with all free parameters at $0.2<z \leq 1.7$, and with $\alpha$ set to evolve at $1.7<z \leq 4.5$. They are reported in Table 1, and the corresponding LFs are plotted all together in Fig. 2. They are used to derive the luminosity densities (Sect. 4) and the star formation rate densities (Sect. 5). At $z>1.7$, the uncertainties of these densities will include the possible span of $\alpha$ between the values -1.1 and -1.73 .

\subsection{Main features of the derived FUV LF}

Our results show a constant and flat $(\alpha \simeq-1)$ FUV faint-end slope at $z<2$, while we set it to steepen with $z$ at $z>2$, where our data can not constrain it. We find that $M^{*}$ brightens monotonically with increasing redshift for the entire redshift range explored. Conversely, at $z \gtrsim 0.9 \phi^{*}$ starts decreasing, with a remarkable drop especially at $z \gtrsim 2$. These trends are evident in Fig. 2 and in Table 1, and are also summarised in Fig. 8.

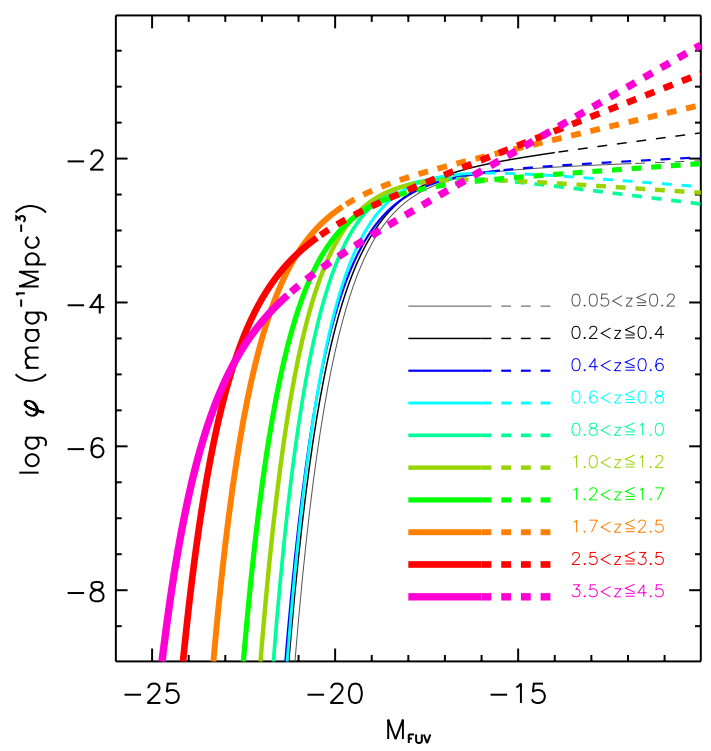

Fig. 2. The VVDS rest-frame FUV-band luminosity functions from $z \simeq$ 0.1 up to $z \simeq 4$ (from the thinnest to the thickest curve), fitted with a Schechter functional form parametrised with the Schechter parameters $\left(\alpha, \phi^{*}, M^{*}\right)$ reported in Table 1 . Lines are dashed for magnitudes fainter than the LF magnitude bias in each redshift bin (see Sect. 3.1).

\subsubsection{A persistent flat faint-end slope at $z<2$}

Table B.2 lists $\alpha$ values used in the literature. Contrarily to our work, at $z<2$ most previous studies were not faint enough to determine the faint-end slope, which was then often set to values as different as $-1.1<\alpha<-1.6$. Nevertheless, for instance, Arnouts et al. (2005) and Oesch et al. (2010) estimated a steep, but also constant, FUV faint-end slope $\alpha \simeq-1.6$ at $0.4<z<1.2$ and $\alpha=-1.7$ at $0.75<z<2.5$, respectively. In contrast, low- $z$ 
values $(z<0.4)$ are found rather flat $\alpha \simeq-1.2$ (e.g., Arnouts et al. 2005; Wyder et al. 2005). To establish the robustness of our results, Appendix B details our three tests summarised below.

For the first test, we derived the rest-frame FUV $1 / V_{\max }$ estimates using the VVDS photometric redshift catalogue, $1.25 \mathrm{mag}$ deeper than the VVDS spectroscopic catalogue (i.e., $I_{A B}=26$ ), over the same VVDS area for a total of $\sim 43000$ sources (see Fig. B.1). A similar flat faint-end slope is found. Thus our derived flat slopes are not due to a large amount of faint blue galaxies missing in the spectroscopic sample, or to an inadequate weighting scheme. With this multi-wavelength $\left(u^{*} g^{\prime} r^{\prime} i^{\prime} z^{\prime} J H K_{\mathrm{s}}\right)$ catalogue, we also tested the effect of selecting galaxies upon their observed $u^{*}$-band up to 26 mag (i.e., based on a rest-frame ultraviolet selection at $z<1$ ) rather than the VVDS observed $I$-band selection (i.e., based on a rest-frame optical selection at $z<1)$. Even though the faint-end slope of such a sample is slightly steeper, but not by more than 0.1 , it stays very constant and close to flatness at $z \lesssim 1$. Thus, our $I$-band selection is unlikely missing a significant population of rest-frame blue galaxies, that could have caused an hypothetic steeper slope.

In the second test, we confirm that the blue, or very blue galaxy population of the VVDS I-selected spectroscopic survey, does not give a steeper slope than the global galaxy population (see Fig. B.2). In rest-frame bands redder than UV (e.g. $U, B)$, the shape of the LF is highly dependent upon the type of galaxies. For instance, in Zucca et al. (2006) at $0.4<z<0.9$, we found $\alpha \simeq-0.9(/-1)$ in $U(/ B)$-band for the blue galaxy population, $\alpha \simeq-1.7(/-1.6)$ in $U(/ B)$-band for the very blue galaxy population, and $\alpha \simeq-1.2$ in both $U$ and $B$ bands for the global population. The diversity of the shape occurs because in bands redder than FUV, the radiation is contaminated by a long-lived stellar population, that makes highly distinctive the galaxy spectral energy distributions for different galaxy types. In contrast, the FUV-band radiation is dominated by a single stellar population of short-lived, massive stars, whatever the type of galaxies. This explains why we observe little change in our $\alpha$ FUV slope between the global galaxy population and the blue, or very blue, galaxy populations. Another possible additional effect is the rising dust attenuation towards shorter wavelengths (Driver et al. 2008), that increases the dispersion of the restframe $B-F U V$ colours. This dispersion, if dependent on the luminosity, may weaken the $B$-band steep slope when using the same galaxy sample to compute the LF in $F U V$-band.

In the third test, we derive the rest-frame NUV LFs at $0.05<$ $z \leq 4.5$ (see Fig. B.3 and Table B.1). The faint-end slope is also flat at $z \lesssim 1.5$ also in the NUV band, showing that the flatness of our FUV slope is not due to possible problems induced by our template extrapolations at $z<1$.

In summary, the robustness of our flat UV faint-end slope is well-established at $z<2$, and it is more consistent with the faint-end slope the local universe, i.e. $\alpha \simeq-1.2$ (Wyder et al. 2005). Despite its low value, we will see (Sect. 5.2) that our dustcorrected SFRD using our rest-frame FUV LFs fully agrees with other measurements at $z<1$, a cosmic period where the SFRD seems now well-constrained with different SFR estimators. This is because we correct the FUV radiation for dust attenuation in an appropriate way as we describe later.

\subsubsection{Comparing FUV LFs at $z>2$}

At $z>2, M_{\mathrm{FUV}}^{*}$ keeps on brightening, while $\phi^{*}$ drops. Thanks to the depth of our data, these trends are robust, whichever the choice of $\alpha$ (see Table B.1).
We remark that the drop in $\phi^{*}$ that we see at $z>2$ is likely unrelated to incompleteness of low surface brightness objects, although this effect can not be completely ruled out. As mentioned in Sect. 2.1, the VVDS survey photometric catalogue, down to $I_{A B} \sim 24.75$, is $90(/ 70) \%$ complete in observed surface brightness down to 24.5(/25) mag/arcsec ${ }^{2}$. As discussed in McCracken et al. (2003, Sect. 4.1), objects with particularly low surface brightness, that could fall below our detection limit at high redshift, are very few, while "normal" galaxies would be in any case detected. Still, a possible concern can be raised if we consider that the above-mentioned observed surface brightness limits correspond to brighter and brighter absolute limits going to high redshift. We will see (Sect. 5.3) that the major contribution to the total SFRD at $z>3$ is given by very luminous galaxies, so in principle our results at these epochs should be driven by galaxies with an absolute surface brightness bright enough to be within the detection limits. Nevertheless, we could miss a population of low absolute surface brightness galaxies, for which we would not be complete at high redshift. We performed a very simple test to quantify, at a first order of magnitude, this possible effect. We considered the redshift bin $3.5<z<4.5$, where the I-band filter, used to select VVDS galaxies, corresponds to the rest-frame FUV light. This way, to pass from the surface brightness in $I$-band shown in McCracken et al. (2003) to an absolute FUV-band surface brightness we can neglect $K-$ and colourcorrections. We verified that all the galaxies brighter than the Deep $^{2}$ LF bias $\left(M_{\mathrm{FUV}}=-22\right)$ have an absolute surface brightness brighter than $18 \mathrm{mag} / \operatorname{arcsec}^{2}$, that coresponds, at $z \sim 4$, to the observed limit of $25 \mathrm{mag} / \operatorname{arcsec}^{2}$ (considering only the cosmological dimming, with no $K$ - or colour-corrections), and only a small fraction is fainter than $17.5 \mathrm{mag} / \mathrm{arcsec}^{2}$, corresponding at $z \sim 4$ to the observed limit of $24.5 \mathrm{mag} / \operatorname{arcsec}^{2}$. In summary, even if small effects can not be completely ruled out, we believe that our results are robust with respect to surface brightness completeness.

We also verified that the low $\phi^{*}$ value at $z>2$ is robust with respect to the weighting scheme (see Appendix A) that we apply to the spectroscopic sample. Thanks to this weighting scheme, we are indeed able to properly recover the total galaxy population in the photometric catalogue. Figure B.1 shows the FUVband LFs computed using the entire photometric catalogue (with photometric redshifts): it overlaps with the LFs computed using the VVDS Deep+Ultra-Deep spectroscopic sample, reinforcing the reliability of our weighting scheme.

Table B. 2 shows clearly that the determination of $\alpha$ is a major source of uncertainty, and even when it is fitted and not set, it can be as flat as $\sim-1.2$ or as steep as $\sim-1.7$. The scatter on $\alpha$ is accompanied by analogous ones for $M_{\mathrm{FUV}}^{*}$ and $\phi^{*}$. We verified that our $M_{\mathrm{FUV}}^{*}$ is generally brighter than other works in literature, at all $z=2,3,4$, while our $\phi^{*}$ is much smaller, especially at $z=3$ and 4 (see e.g. Gabasch et al. 2004, at $z=2,3$; Steidel et al. 1999, at $z=3,4$; Arnouts et al. 2005, at $z=3$; Bouwens et al. 2007, at $z=4$; Reddy \& Steidel 2009, at $z=2,3$; Paltani et al. 2007, at $z=3.5$; Gabasch et al. 2004, at $z=3.5,4.5$; Sawicki \& Thompson 2006, at $z=2,3,4$; Tresse et al. 2007, at $z=3,3.6,4.3)$. We remark, however, that in a few cases we are in agreement with some previous work for what concerns $M_{\mathrm{FUV}}^{*}$ and/or $\phi^{*}$ : we agree on both $M_{\mathrm{FUV}}^{*}$ and $\phi^{*}$ with Gabasch et al. (2004) at $z=2.5$ and with Oesch et al. (2010) at $z \sim 2$, and we

\footnotetext{
2 In this redshift range, only a very small fraction of the galaxies that we use to compute the LF are from the Ultra-Deep sample, as can be seen in Fig. A.2.
} 
agree on $M_{\mathrm{FUV}}^{*}$ with Paltani et al. (2007) at $z=3.5$ and Arnouts et al. (2005) at $z \sim 3$.

This comparison cannot be exhaustive, because it is hard to directly compare the LF shapes from different works. This happens for the following reasons. First, the three Schechter parameters are linked together; therefore, setting $\alpha$ will in some sense determine also $M^{*}$ and $\phi^{*}$, and we already showed that $\alpha$ have been set, or determined, to very different values. Second, computing the Schechter parameters fitting the $1 / V_{\max }$ values or using the STY method can lead to slightly different results. Third, different FUV-band filters or central wavelength have been used in the literature (peaked at $1350 \leq \AA \leq 1700$, see Table B.2).

As a final remark about comparing LFs in the literature, we detail in Appendix $\mathrm{C}$ the difficulty in comparing FUV luminosity functions directly-observed (as ours) with those derived from FUV number counts (as in Steidel et al. 1999, for instance). With this analysis, we explain the following remarkable evidence: at $2.7 \leq z \leq 3.4$, on one side Le Fèvre et al. (2005a) and Le Fèvre et al. (in prep.) show that the VVDS presents surface number counts (per apparent magnitude) at least two times larger than those found by Steidel et al. (1999), and this is particularly evident for bright galaxies $\left(I_{A B} \lesssim 23.5\right)$; on the other side, the VVDS rest-frame FUV LF is only $\sim 50 \%$ higher at bright magnitudes than the one found by Steidel et al. (1999). We refer the reader to Appendix $\mathrm{C}$ for details. The conclusion of this exercise is that number counts, in colour-selected samples, are less representative of the complete galaxy population than those in flux-limited samples, as the respective $n(z)$ of these samples are very different. This exercise also confirms that one can not easily transform number counts to LF in the case of a skewed $n(z)$.

\section{The FUV comoving luminosity densities}

We derive the mean comoving luminosity density (LD) in each redshift bin as $\mathrm{LD}=\int_{L_{\text {faint }}}^{L_{\text {brigt }}} \phi(L) L \mathrm{~d} L$, where $\phi(L)$ is the luminosity function assuming a Schechter (1976) functional form as done in Sect. 3. We set $L_{\text {faint }}=10^{15} \mathrm{~W} \mathrm{~Hz}^{-1}$ and $L_{\text {bright }}=$ $10^{25} \mathrm{~W} \mathrm{~Hz}^{-1}$ (corresponding to $M_{\text {faint }} \sim-3.4$ and $M_{\text {bright }} \sim$ -28.4 ), to adopt the same limits as in the compilation of Hopkins (2004) and to allow an easier comparison with the literature. Given the typical shape of the LF (steeply decreasing for bright galaxies), setting $L_{\text {bright }}$ as above or $L_{\text {bright }}=\infty$ does not make any difference. Setting the faint limit at $L_{\text {faint }}=10^{15} \mathrm{~W} \mathrm{~Hz}^{-1}$ gives a total LD on average $0.3 \%$ different from the definition with $L_{\text {faint }}=0$.

\subsection{The VVDS FUV comoving luminosity density}

The left panel of Fig. 3 shows the FUV-band LD that we derived from our best LF determinations at $0<z<4.5$ and tabulated with their total uncertainties in Table 1. Our LD uncertainties include errors from the STY LF fit, errors due to cosmic variance, Poisson noise, and errors associated to our weighting scheme. The relative uncertainties induced on the total LD by each different source of error are listed in Table 2.

Errors derived from the STY estimator are underestimated at $z>1.7$ since $\alpha$ has been fixed in these redshift bins. In these cases, we computed the LD also assuming the two extreme values of $\alpha(-1.1,-1.73)$, and the percentage difference with our best LDs at $z>1.7$ was added in quadrature to the STY errors. Cosmic variance $(\mathrm{CV})$ errors are computed using the recipe in
Driver \& Robotham (2010). We computed CV errors also with the recipe in Trenti \& Stiavelli (2008), and we found lower estimates at $z<1.2$, going from $\sim 20 \%$ at $z=0.1$ down to $\sim 12 \%$ at $z=1.1$. The same computation based on Somerville et al. (2004) gives results similar to those by Trenti \& Stiavelli (2008). We decided to use the more conservative values found with the recipe in Driver \& Robotham (2010). Errors associated to the weighting scheme are computed as follows. The weight for each galaxy has an error derived in propagating the Poisson noise in the weighting formulas detailed in Appendix A. We computed again the LFs using the weights plus and minus this error, and we derived the maximal and minimal LDs. Their percentage difference with respect to the best LDs gives errors arising from the weights. This error is negligible below $z \sim 1.7$. In each redshift bin, the final LD uncertainty is the addition in quadrature of all these sources of errors. From Table 2, it is evident that CV errors dominate at $z<1.7$, while at $z>1.7$ the total uncertainty is dominated by errors from the weighting scheme and by the percentage difference when assuming the two extreme values of $\alpha$ with respect to the chosen $\alpha$ value. It is worth noticing that our largest uncertainty, the upper error bar at $z \sim 2$, is given mainly by the uncertainty on the value of $\alpha$ : it includes the difference between the LD with our best $\alpha(=-1.3)$ and the extreme value $\alpha=-1.73$. We remind the reader that such extreme $\alpha$ value, that we think is not optimal for our data at this redshift, nevertheless has been found in the literature (Reddy \& Steidel 2009; Oesch et al. 2010) at very close redshift. Other findings from other works span at this $z$ the range $-1.7 \lessgtr \alpha \lesssim-1.1$. In Table B.1 we give a complete list of the LDs for our LFs described in Sect. 3.2, i.e. including the best LFs, those with all free STY parameters at $z \sim 0.1$ and $z>1.7$, and with $\alpha$ set to -1.1 or -1.73 at $z>1.7$.

In the literature the treatment of cosmic variance as a source of uncertainty differs from work to work. If we consider the data sets overplotted in Figs. 3 and 5, we note the following. Some authors do not take into account the role of cosmic variance (e.g., Schiminovich et al. 2005; Wilson et al. 2002; Iwata et al. 2007); or consider it negligible with respect to statistical uncertainties (e.g., Paltani et al. 2007); or consider it negligible because of the large volume explored (e.g., van der Burg et al. 2010, $4 \mathrm{deg}^{2}$ ); or mitigate its effects using several fields in different lines of sights, although generally these fields are much smaller than the VVDS2h field (e.g., Steidel et al. 1999; Reddy \& Steidel 2009); or the cosmic variance has been included as uncertainty in the LF normalisation (e.g., Bouwens et al. 2007). We choose to compute it with the most conservative method (see above) and to include it in our comprehensive uncertainty on the LD.

The right panel of Fig. 3 shows the FUV-band LDs for galaxies brighter than $L^{*}$ at $z \sim 0$, i.e., the determination by Wyder et al. (2005), corresponding to $M^{*}=-18.12$. In Fig. 3, we overplot data points of other studies which published the three Schechter parameters of their rest-frame ultraviolet LFs, that we integrated also to $L_{\text {faint }}=10^{15} \mathrm{~W} \mathrm{~Hz}^{-1}$ (left panel) and to $L_{\text {faint }}=L^{*}(z=0)$ (right panel).

From the left panel in Fig. 3, it is evident that the VVDS LD derived in this work shows a peak in its evolution with time. After an increase by a factor of $\sim 2$ from $z \sim 4$ to $z \sim 2$, the LD decreases sharply by a factor of $\sim 4.5$ down to $z \sim 0$. Figure 2 shows that the LD is increasing from $z \sim 0$ to $z \sim 2$ because of the continuous brightening of $L^{*}$, with the normalisation and the faint end slope not changing so much. The brightening of $L^{*}$ also assures that the LD at $z \sim 2$ would be robustly larger than 


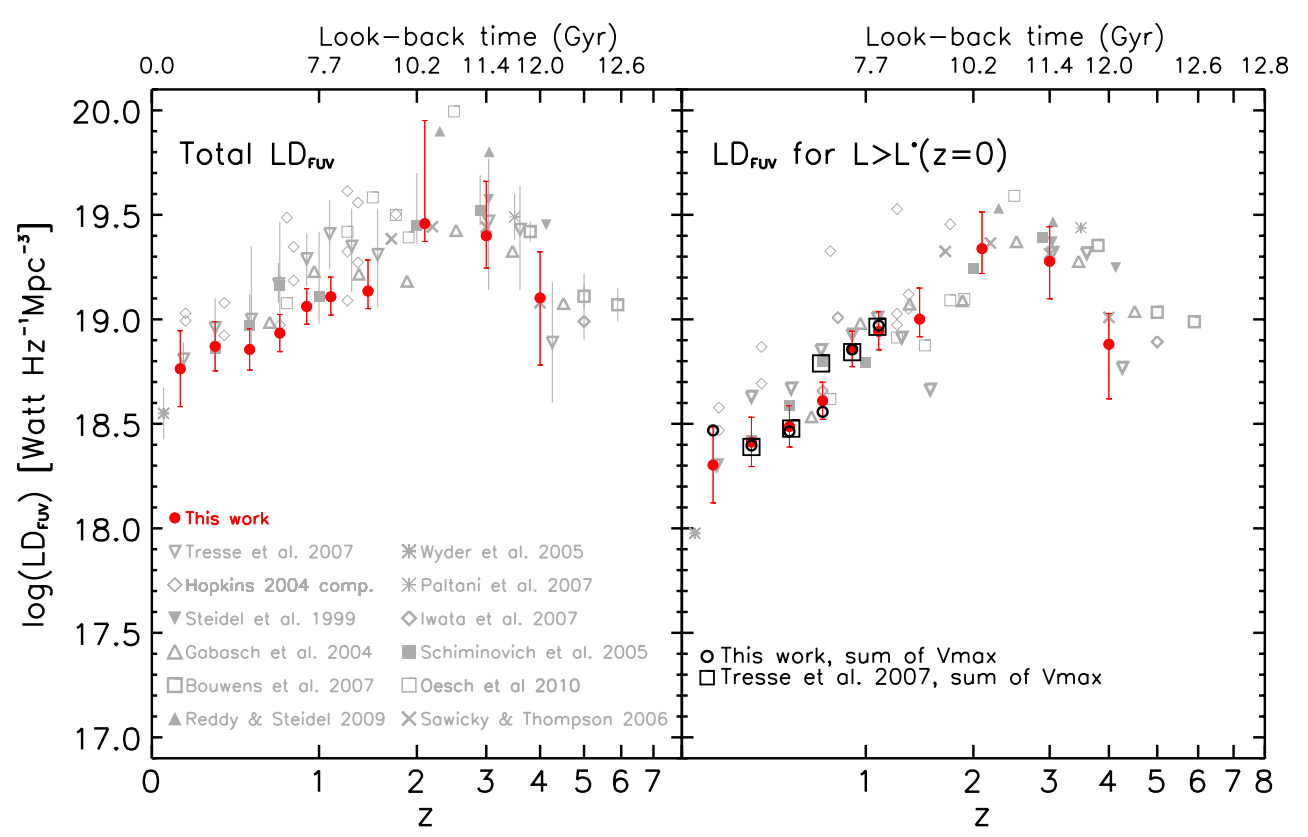

Fig. 3. Left panel. VVDS Deep+Ultra-Deep FUV-band luminosity densities derived from our best LF determination (FUV-150 red filled circles) as a function of redshift. Error bars are explained in Sect. 4. Gray data points are ultraviolet LF estimates found in the literature, as detailed in the labels (see also at the end of the caption). Right panel. FUV-band luminosity densities restricted to galaxies brighter than $L^{*}$ at $z=0$ in Wyder et al. $(2005)\left(M_{\mathrm{FUV}}=-18.12\right)$. Symbols are like in the left panel. Black open circles and squares represent the LD down to $M_{\mathrm{FUV}}=-18.12$ summing the $1 / V_{\max }$ points in this work and in Tresse et al. (2007), respectively, instead of integrating the Schechter LF fit. We did this only up to $z \sim 1$ because in VVDS data at $z \gtrsim 1$ the bias limit for $1 / V_{\max }$ computation is brighter than $M_{\mathrm{FUV}}=-18.12$. List of references: Tresse et al. (2007) (VVDS Deep, FUV-150, empty upside-down triangles); compilation of FUV and NUV data extracted from Hopkins (2004) (thin open diamonds); Steidel et al. (1999) (FUV-1700, filled upside-down triangles); Schiminovich et al. (2005) (FUV-150, filled squares those at $z<1.2$ GALEX-VVDS for $z<1.2$ data); Bouwens et al. (2007) (FUV-1600, thick open squares); Reddy \& Steidel (2009) (FUV-1700, filled triangles); Wyder et al. (2005) (GALEX-2dFGRS, FUV-150, thick asterisk); Paltani et al. (2007) (FUV-1700, thin asterisk); Iwata et al. (2007) (FUV-1700, thick open diamonds); Gabasch et al. (2004) (FUV-150, open triangles); Oesch et al. (2010) (FUV-150, thin open squares); Sawicki \& Thompson (2006) (FUV-1700, crosses).

at $z \sim 1.5$, even if at $z \sim 2$ we had used a flat slope ${ }^{3}$. In contrast, at $z>2$ the LD evolution is mainly driven by the evolution of the normalisation: the LD decreases from $z \sim 2$ to $z \sim 4$ because of the steep decrease of $\phi^{*}$. We note also that the decrease of the LD for $z>2$ with our best estimate (red circles) is even smoothed away because we are assuming $\alpha$ increasing with redshift (that should cause the LD to increase). Only by using a much sharper steepening of $\alpha$ at $z>2$ we could make the LD peak disappear, making the LD staying roughly constant at $z>2$. For any other choice (the slope slowly increasing with $z$, or fixed to a flat or steep value like in the two extremes that we consider in the error bars), the LD is clearly decreasing at $z>2$.

Our result is an improvement with respect to the similar study of the FUV-band LD in Tresse et al. (2007), that was based on our first VVDS-Deep spectroscopic sample (Le Fèvre et al. 2005b) and our UBVRI photometric sample (Le Fèvre et al. 2004b). On one side, the so-called redshift desert $(2<z<3)$ could not be analysed, as it is now thanks to our Ultra-Deep data. On the other side, the spectroscopic and photometric data were too shallow to determine $\alpha$ of the FUV-band LF, and thus a constant value $(\alpha=-1.6)$ was used over the explored redshift range $(0<z<2,2.70<z<5)$, as previously done in the literature (see Table B.2). Thanks to our new spectroscopic and photometric data, with the present work it is the first time that the presence of a peak at $z \sim 2$ in the FUV-band LD evolution has been robustly assessed from a single survey.

\footnotetext{
3 We remind that the lower limits of the error bars at $z>1.7$ include the possibility of $\alpha=-1.1$.
}

Table 2. Relative uncertainty to the total LD (sixth column of Table 1) from different sources of error, expressed in percentage.

\begin{tabular}{|c|c|c|c|c|c|}
\hline$\Delta z^{a}$ & $\mathrm{STY}^{b}$ & $\begin{array}{r}\alpha \text { range }^{c} \\
\text { err }\end{array}$ & $\begin{array}{l}\mathrm{Po}^{d} \\
\text { in \% }\end{array}$ & $\mathrm{CV}^{e}$ & $w^{f}$ \\
\hline $0.05-0.2$ & $\begin{array}{l}+10.9 \\
-9.8\end{array}$ & - & 6.3 & 40 & $\sim 0$ \\
\hline $0.2-0.4$ & $\begin{array}{l}+7.9 \\
-6.5\end{array}$ & - & 3.0 & 26 & $\sim 0$ \\
\hline $0.4-0.6$ & $\begin{array}{l}+5.0 \\
-4.4\end{array}$ & - & 2.9 & 22 & $\sim 0$ \\
\hline $0.6-0.8$ & $\begin{array}{l}+3.7 \\
-3.3\end{array}$ & - & 2.6 & 20 & $\sim 0$ \\
\hline $0.8-1.0$ & $\begin{array}{l}+3.9 \\
-3.4\end{array}$ & - & 2.8 & 19 & $\sim 0$ \\
\hline $1.0-1.2$ & $\begin{array}{l}+9.7 \\
-6.4\end{array}$ & - & 3.4 & 19 & $\sim 0$ \\
\hline $1.2-1.7$ & $\begin{array}{l}+32 \\
-15\end{array}$ & - & 3.4 & 12 & $\sim 0$ \\
\hline $1.7-2.5$ & $\begin{array}{l}+5.5 \\
-4.9\end{array}$ & $\begin{array}{l}+112 \\
-12\end{array}$ & 7.2 & 10 & $\begin{array}{l}+11.3 \\
{ }_{-7.8}\end{array}$ \\
\hline $2.5-3.5$ & $\begin{array}{l}+3.5 \\
-3.3\end{array}$ & $\begin{array}{l}+56 \\
-32\end{array}$ & 5.8 & 9 & $\begin{array}{l}+17.0 \\
-9.6\end{array}$ \\
\hline $3.5-4.5$ & $\begin{array}{l}+7.3 \\
-6.4\end{array}$ & $\begin{array}{l}+0 \\
-55\end{array}$ & 8.8 & 10 & $\begin{array}{l}+48.4 \\
-46.9\end{array}$ \\
\hline
\end{tabular}

Notes. Their sum in quadrature give the total error quoted in Table 1. For details on the computation of each error see Sect. 4.1. ${ }^{(a)}$ Redshift bins. ${ }^{(b)}$ Relative error induced by the STY LF fit. ${ }^{(c)}$ Relative uncertainty derived from the difference in the total LD between the "best" $\alpha$ value quoted in Table 1 and the lower $(\alpha=-1.1)$ and upper $(\alpha=-1.73)$ limits considered. It is considered only when $\alpha$ is set. ${ }^{(d)}$ Relative error induced by Poisson noise. ${ }^{(e)}$ Relative error induced by cosmic variance. (f) Relative error derived accounting for the error of the galaxy weighting scheme in the LF fit. 


\subsection{Discussion and comparison with literature}

A large scatter between the different LD determinations is present in both panels of Fig. 3. In integrating a Schechter LF down to the faintest luminosities, the value of the faint-end slope has a strong impact, especially if it is steep, leading to very different LDs for different $\alpha$ (see, e.g., Fig. 1 in Tresse et al. 2007). Table B. 2 shows that the $\alpha$ determinations span a large range of values at every redshifts, and it is evident that our determinations of $\alpha$ are lower than the average, especially at $z<1.7$. This is the main reason why our total LD appears on average lower than the other values in the literature (but still in agreement with works that used $\alpha \simeq-1$, like in Gabasch et al. 2004). We have already discussed in Sect. 3 the difficulty to compare rest-frame FUV LFs, and thus LDs, from various surveys, contrarily to rest-frame optical LFs and LDs. By integrating the LFs down to $M_{\mathrm{FUV}}=-18.12$ (right panel of Fig. 3), the differences in the values of $\alpha$ should have little impact on the resulting LD, when this integration limit is very close to $M^{*}$ (i.e. at $z<1$ ). The observed scatter in the right panel of Fig. 3 means that $L^{*}$ and $\phi^{*}$ are also discrepant among various data sets. Furthermore, since the three Schechter parameters $\left(L^{*}, \phi^{*}, \alpha\right)$ are strongly correlated, different values of $\left(L^{*}, \phi *\right)$ arise even within the same data set when $\alpha$ is fixed to different values (see below). For instance, taking a steeper $\alpha$ leads to a fainter $L^{*}$ and a lower $\phi *$ to fit exactly the same data set.

In the right panel of Fig. 3 we also show our $L>L_{z=0}^{*}$ LDs obtained by summing the $1 / V_{\max }$ values for galaxies with $L_{z=0}^{*} \leq L \leq L_{\text {bright }}$ (black empty circles). The discrepancies between this estimate and the one using the integration of the Schechter function mirror the level of extrapolation made using a STY fit. We can see that, within the error bars, our extrapolation is small, which ensures the goodness of our fit at $M_{\mathrm{FUV}}<-18.2$. The same exercise is repeated for the data in Tresse et al. (2007) (black empty squares).

We can test the effect of different LF parameterisations on total LD estimates, using two works within the VVDS survey. Tresse et al. (2007) used the VVDS Deep data presented in Le Fèvre et al. (2005b) ( $U B V R I)$ to derive the rest-frame FUV150 LDs. Their slope $\alpha$ has been set to -1.6 , based on GALEXVVDS results from Arnouts et al. (2005) at $0.2<z<1$, since the depth of the $U$-band data available at that time did not enable them to constrain the faint-end slope as we can do with our new $u^{*} g^{\prime} r^{\prime} i^{\prime} z J H K_{\mathrm{s}}$ data. Therefore, their LDs at $z \lesssim 1.5$ are higher than those found in this work for both the total galaxy sample and the $L>L_{z=0}^{*}$ galaxy sample. Nevertheless, if one compares the minimal LDs (i.e., the sum of the $1 / V_{\max }$ ) between this work and Tresse et al. (2007), they are in fairly good agreement.

Another example is given by the total LDs from Reddy \& Steidel (2009) at $z \sim 2$ and $\sim 3$, that are a factor of $\sim 2.5$ larger than ours. The difference is mainly explained by the much steeper slope in their LF (see Table B.2). In fact, from the left panel of Fig. 3 we see that their LD is in much better agreement with the upper limits of our error bars, that correspond to the extreme case with $\alpha=-1.73$.

It is worth noticing that Oesch et al. (2010) find a constant $\alpha$ within $0.5<z<2.5$, but much steeper than ours $(\alpha \sim-1.7)$. Moreover, they do find a peak in the LD evolution as we do, but at $z \sim 2.5$. We remark that they have to use points from the literature to find this peak (the highest $z$ of their LD determinations being $z=2.5$ ), while we are able to constrain the LD peak using our own homogeneous data.

More generally, comparison with literature appears tricky, not only because of very different assumptions on the LF shape (the need to set $\alpha$ or $M^{*}$, for instance), but also because the LF shape can be intrinsically different according to the various survey characteristics (flux limit, band of selection...). Due to this difficulty in comparing different works, it is clear the importance of tracing the rest-frame FUV LDs over a wide range of redshifts with a single and homogeneous survey. This is what we have been able to do with our data set.

\section{The dust attenuation and the star formation rate densities up to $z=4.5$}

A robust determination of the dust attenuation is a critical element to transform the luminosity density into the effective star formation rate density. Thanks to our present VVDS Deep+Ultra-Deep spectroscopic sample, combined with a wide range of deep multi-wavelength data $\left(u^{*} g^{\prime} r^{\prime} i^{\prime} z J H K_{\mathrm{s}}\right)$, we can now study in details and with homogeneity the cosmic dust attenuation evolution over $0<z<4.5$.

In the following subsections, we explain how we determined the dust attenuation in our sample and how it compares with (and improves upon) other measurements in the literature (Sect. 5.1). Then, we show how we derived the dust-corrected SFRD for the global galaxy population (Sect. 5.2), and how galaxies with different luminosities contribute to the total SFRD (Sect. 5.3).

\subsection{The dust attenuation}

Estimating the average dust attenuation properties in a galaxy survey is a complex task. A number of authors have suggested various methods to derive the dust attenuation using a multiwavelength data set. This leads to one important source of scatter in the literature in the determination of the dust corrected SFRDs. For instance, dust attenuation can be estimated using dust reddening curves or UV slopes in the FUV, or the Balmer decrement measured from $\mathrm{H} \alpha$ and $\mathrm{H} \beta$ (with the drawback that $\mathrm{H} \alpha$ is difficult to observe for large samples at $z>0.3$ ). It can be also derived with multi-wavelength SED fitting techniques, especially when the knowledge of spectroscopic redshifts enables to break a dust-redshift degeneracy. Nevertheless, if there is a large and prominent population of highly dust-obscured galaxies, it will be missed by deep rest-frame FUV data sets. In principle, a powerful method is based on adding the total SFR radiation, that is the one passing through dust (FUV emission) and the one re-emitted by dust (FIR emission). However, this method has potential drawbacks. For example, results will be exclusively linked to those galaxies visible at both wavelengths, and mainly at $z<2$. Furthermore, the situation in infrared survey observations is not optimal due to unknown contents of cold and hot dust, or broad-line active galaxy nuclei contamination, or large PSFs making difficult the measurement of uncontaminated single sources. We refer the reader to the discussion in Calzetti (2008) for details on computing dust attenuation using both FIR and FUV data.

Since we want to derive the dust attenuation in a similar manner over $0<z<4.5$, we estimate the dust attenuation using the results from the SED fitting analysis. This method is not always fully reliable on a object-by-object basis. Still, it provides a good estimate of the average attenuation properties of the whole population under study and of their evolution with time. Also it avoids the use of restricted cross-matched optical-FIR catalogues.

We primarily use the recipe in Calzetti et al. (2000) for actively star-forming galaxies to derive our dust attenuations at $1500 \AA$, and we also report and discuss those derived with other 


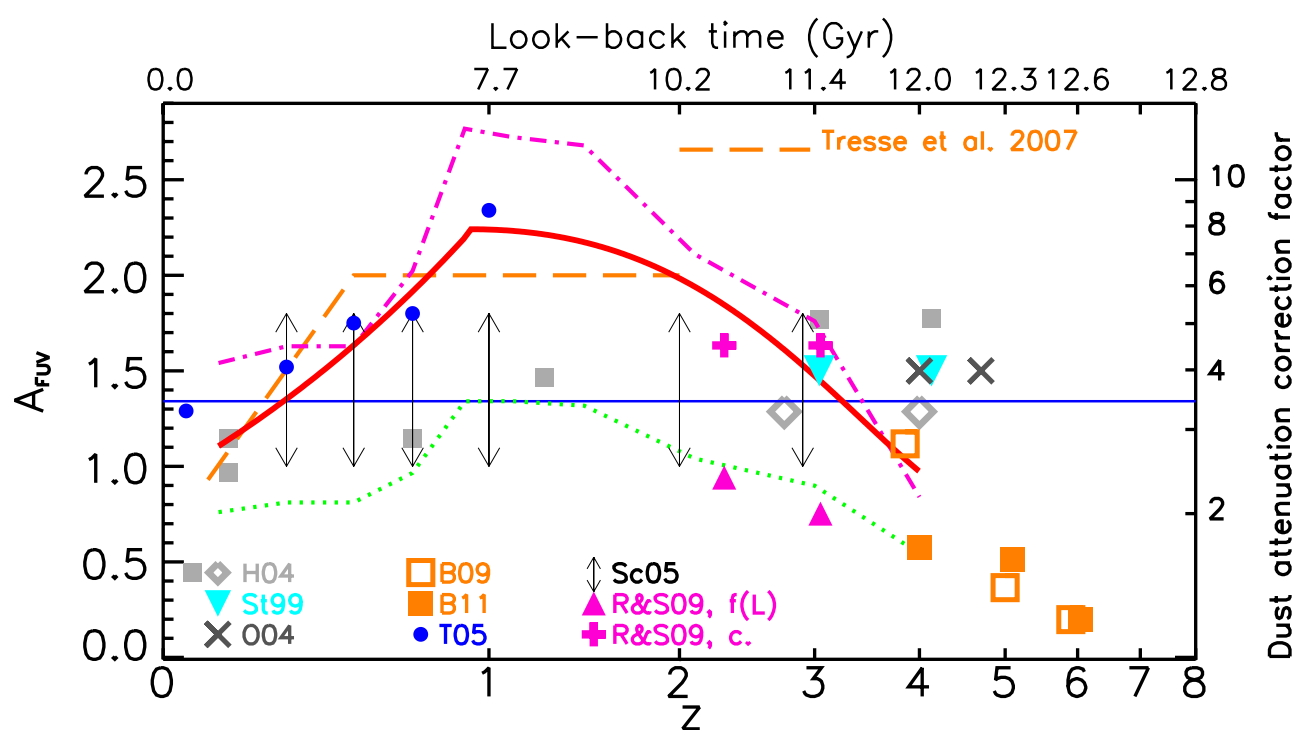

Fig. 4. Dust attenuation $A_{\mathrm{FUV}}$ in magnitudes as a function of redshift. The thick solid red curve represent the fit (see text for details) of the average dust attenuation, as a function of redshift, determined in this work, using the Calzetti's law. The $A_{\mathrm{Fuv}}$ values derived from this fit in each redshift bin are listed in Table 1. The magenta dot-dashed curve is the average $A_{\mathrm{FuV}}$ determined in this work using the recipe in Meurer et al. (1999), based on the $\beta$ slope. The green dotted curve is the same as the dot-dashed one, but here we use the recipe in Cortese et al. (2006), calibrated with normal star forming galaxies. The thin blue horizontal solid line is a constant $A_{\mathrm{FUV}}$ computed with the Calzetti's law using one single typical value of $E(B-V)(=0.13)$. The right $y$-axis shows the multiplicative factor to be applied to the observed luminosities, i.e., $10^{0.4 A_{\mathrm{Fuv}}}$. All the other symbols represent dust attenuations found in literature, as indicated in the labels: orange dashed line from Tresse et al. (2007) (obtained comparing notcorrected SFRDs with dust-corrected SFRDs from other works, see text for details); gray open diamonds and filled squares from the compilation in Hopkins (2004, H04) (only FUV and NUV determinations, diamonds and squares for a correction independent from or dependent on SFR, respectively); cyan filled upside-down triangles from Steidel et al. (1999, St99); thick gray crosses from Ouchi et al. (2004, O04); orange open and filled squares from Bouwens et al. (2009, B09) and Bouwens et al. (2011, B11) respectively (slightly shifted in redshift for clarity); blue filled circles from Takeuchi et al. (2005, T05), based on the 02h field GALEX-VVDS LFs (Arnouts et al. 2005) and the CDFS field Spitzer 15- $\mu$ m LFs (Le Floc'h et al. 2005) at $0.2<z<1$; arrows from Schiminovich et al. (2005, Sc05), also based on GALEX-VVDS survey; filled magenta triangles and magenta crosses from Reddy \& Steidel (2009, R\&S09) (triangles and crosses for a dust attenuation dependent on or independent from FUV luminosity, respectively).

recipes. We follow the prescription in Calzetti et al. (2000, see Eq. (4)), that is, $A(\lambda)=E(B-V)_{\text {star }} k(\lambda)$, where $E(B-V)_{\text {star }}$ is the intrinsic colour excess of the stellar continuum of a galaxy, and $k(\lambda)$ is the starburst reddening curve in Calzetti et al. $(2000)^{4}$ at $[0.12-0.63] \mu \mathrm{m}$. As described in Sect. 2.2, our template SED fitting assigns to each galaxy a value of $E(B-V)_{\text {star }}$ chosen from a grid of five possible values $(0.1,0.2,0.3,0.4,0.5)$. In each studied redshift bin, we compute our resulting mean $E(B-V)_{\text {star }}$ and $A_{\mathrm{FUV}}$. We remark that our $E(B-V)_{\text {star }}$ computations do not seem to depend on observed (i.e. not dust-corrected) rest-frame FUV luminosities, which indicates that our sample includes a large variety of actively star-forming galaxies at each luminosity. For this reason, we are confident that our mean $E(B-V)_{\text {star }}$ values, obtained averaging galaxies brighter than the given magnitude bias at each redshift, do not depend on the range of luminosity spanned at each redshift.

Figure 4 shows our results. We find that $A_{\mathrm{FUV}}$ increases steeply and fast from $z \sim 4$ to $z \sim 2$, i.e. of 1 mag within only 2 Gyr. It keeps on mildly increasing from $z \sim 2$ to $z \sim 1$ $(\sim 0.2 \mathrm{mag}$ in $2.5 \mathrm{Gyr})$ and then it continuously decreases by $\sim 1.1$ mag within $\sim 8$ Gyr from $z \sim 1$ to $z \sim 0$. This results in a maximum dust attenuation value at $z \sim 1\left(A_{\mathrm{FUV}} \simeq 2.2\right)$. We smooth $A_{\mathrm{FUV}}(z)$ with a linear fit and a 3 rd order polynomial function for the redshift ranges $z<1$ and $z>1$, respectively, to preserve the different trends of $A_{\mathrm{FUV}}(z)$ below and above this redshift. The red solid line in Fig. 4 shows our smoothed $A_{\mathrm{FUV}}$, and we give its values in each redshift bin in Table 1 . We remark

\footnotetext{
$\overline{{ }^{4} k(\lambda)=2.659 \times\left(-2.156+1.509 / \lambda-0.198 / \lambda^{2}+0.011 / \lambda^{3}\right)+4.03 .}$
}

that the chosen fits depart from the fitted data by a very small amount, so we do not overplot the original $A_{\mathrm{FUV}}$ for clarity.

We also compute our average $A_{\mathrm{FUV}}$ with the recipe given by Meurer et al. (1999), based also on actively star-forming galaxies, but using the $\beta$ slope of the UV continuum as a proxy to the UV attenuation (dot-dashed magenta line in Fig. 4). We use the $\beta$ expression of Kong et al. (2004) which accounts for the star formation history to reduce the large scatter of this method. Using the $\beta$ slope leads to larger $A_{\mathrm{FUV}}$, in particular at $1 \lesssim z \lesssim 2$. Treyer et al. (2007) show that this method works well at least up to $z=1$, but breaks down for red-sequence and very blue compact galaxies and for the majority of high- $z$ Lyman break galaxies, which form a low-attenuation sequence of their own. Also, Wijesinghe et al. (2011) emphasise that this method overestimates SFRs unless a modified relation between $\beta$ and the attenuation factor is used as indicated by other galactic properties than the UV continuum, in particular for high- $z$ galaxy SFRs. Therefore, a good knowledge of the galaxy population under study is necessary for a reliable application of this method. For instance, we compute our average values of $A_{\mathrm{FUV}}$ with the $\beta$ expression given by Cortese et al. (2006), calibrated on normal star-forming galaxies, and we plotted them in Fig. 4 as well (green dotted line). While this relation follows the same trend as the previous estimates, the resulting attenuations are lower by more than $1 \mathrm{mag}$. On the basis of this analysis, we conclude that taking the Calzetti et al. (2000) reddening curve appears the best solution for the VVDS Deep+Ultra-Deep sample since it is largely dominated by actively star-forming galaxies (from $\sim 40 \%$ at $z \sim 0.1$ up to $\sim 85 \%$ at $z \sim 4.0$, see Appendix B). 
Nevertheless, whatever the method to compute the dust attenuation, the amount of dust increases from $z=4$ to $z=1$, and then decreases down to $z \sim 0$.

In Fig. 4 we also overplot $A_{\text {FUV }}$ dust attenuation values taken from the literature. We can see that data are diversely spread between 1 and $1.8 \mathrm{mag}$. Actually, they are often derived in different ways, depending sometimes on FUV-band luminosity (Reddy \& Steidel 2009), or on SFR (Hopkins 2004), or being a constant value (Schiminovich et al. 2005, GALEX-VVDS). Conversely, Tresse et al. (2007) derived them by comparing the dust corrected $12 \mu \mathrm{m}$ (Pérez-González et al. 2005) and $\mathrm{H} \alpha$ (Tresse \& Maddox 1998; Tresse et al. 2002) SFRDs to their uncorrected FUV-derived VVDS Deep SFRDs. They found that the attenuation at $1500 \AA$ was $\sim 2$ mag from $z=2$ to $z=0.4$, and then it decreased from $z=0.4$ to $z=0$ down to $\sim 1 \mathrm{mag}$. These last $A_{\text {FUV }}$ values are very close to our present work. We plot them in Fig. 4 with an orange dashed line. We are in good agreement also with Takeuchi et al. (2005) at $0.2<z<1$. To estimate the mean dust attenuation, they compared the GALEX-VVDS LFs (Arnouts et al. 2005) to the Chandra Deep Field South Spitzer 15- $\mu$ LFs (Le Floc'h et al. 2005). Our $A_{\mathrm{FUV}}$ agrees also with the one derived by Steidel et al. (1999) at $z \sim 3$ and by Bouwens et al. (2009) at $z \sim 4$ (but see Bouwens et al. 2011, for a much lower $A_{\text {FUV value). }}$

In summary, with the comprehensive VVDS Deep+UltraDeep sample we are able to compute the evolution of the mean cosmic dust attenuation over $\sim 12$ Gyr. It increases rapidly of $1 \mathrm{mag}$ in $2 \mathrm{Gyr}$ (from $z \sim 4$ to $z \sim 2$ ), then it reaches a maximum at $z \sim 1$ (increasing by $\sim 0.2 \mathrm{mag}$ in $2.5 \mathrm{Gyr}$ ), and finally it decreases continuously down to $z \sim 0(\sim 1.1 \mathrm{mag}$ within $\sim 8 \mathrm{Gyr}$. $)$ There is a maximum in dust attenuation at $z \sim 1\left(A_{\mathrm{FUV}} \simeq 2.2\right)$.

\subsection{The dust-corrected SFRD}

To transform FUV fluxes into star formation rates, we use the SFR calibration of Madau et al. (1998). It yields:

$\operatorname{SFRD}(z)=1.4 \times 10^{-28} \operatorname{LD}_{\mathrm{FUV}}(z) 10^{0.4 A_{\mathrm{FUV}}(z)}$,

where the SFRD is in $M_{\odot} \mathrm{yr}^{-1} \mathrm{Mpc}^{-3}$ units and the LD in $\mathrm{erg} \mathrm{s}^{-1} \mathrm{~Hz}^{-1} \mathrm{Mpc}^{-3}$. This formula includes the dust attenuation $A_{\mathrm{FUV}}(z)$, and assumes a Salpeter (1955) initial mass function (IMF) including stars from 0.1 to 125 solar masses. The resulting dust-corrected SFRDs are shown in Fig. 5. The values of uncorrected (i.e., $A_{\mathrm{FUV}}(z)=0$ ) and dust-corrected SFRDs are given in Table 1. For the SFRDs, we assume the same uncertainties as the LDs (see Sect. 3), that is, we do not include any dust reddening errors since it is the choice of the method which dominates (see Sect. 5.1). Still, we include STY fit errors, Poisson noise, cosmic variance and weighting scheme errors. We remind that the STY fit errors, when $\alpha$ is fixed, include the span in LD (and so in SFRD) that one would have setting $\alpha$ at the two extreme values $(-1.1$ and -1.73$)$ discussed in Sect. 3. We overplot in the top panel of Fig. 5 dust-corrected UV-derived SFRDs from other published works, assuming a Salpeter (1955) IMF. We refer to Hopkins \& Beacom (2006) for a detailed comparison among different IMFs.

Like for the LD, we find a peak in the SFRD evolution at $z \sim 2$. This peak is preceded at earlier cosmic epochs by a rapid increase of a factor 6 from $z \sim 4.5$, then followed by a decrease by a factor of 12 to $z \sim 0$. We note that the VVDS Deep+UltraDeep SFRDs are globally, within error bars, in agreement with the literature of UV data sets obtained at various redshifts, but it is the first time that the cosmic SFRD history is continuously traced from $z=0.05$ to $z=4.5$ in a homogeneous way within one sample. We can therefore assess the presence of a peak in the SFRD evolution at $z \sim 2$, without speculating about the different selection functions of disparate surveys ${ }^{5}$. We fitted the SFRD as a function of $z$ below and above $z=2$, using SFRD $\propto(1+z)^{\beta}$. We find $\beta=2.6 \pm 0.4$ at $z<2$, and $\beta=-3.6 \pm 1.9$ at $z>2$. These two fits are shown in the bottom panel of Fig. 5.

We remark that this peak is the result of the combination of the LD evolution and the dust attenuation evolution, but it is not uniquely driven by the dust correction. First of all, we already found a clear peak at $z \sim 2$ in the evolution of the LD. Secondly, the peak of the dust attenuation evolution does not coincide, in terms of time, with the SFRD peak, because we find it at $z \sim$ 1 and not at $z \sim 2$. Surely, the shape of the dust attenuation evolution (steeply decreasing from $z \sim 2$ to $z \sim 4$ ) enhances the relative decrease of the SFRD with respect to the decrease of the LD in the same redshift range, but it is not creating the SFRD peak by itself.

Although the scatter of measurements beyond $z \sim 1$ is increasingly larger, at $z \gtrsim 3$ our measurements are lower than most other measurements. This is mainly due to our use, at $z \sim 3$, of a flatter faint-end slope in the LF than other literature studies, and to a very low $\phi^{*}$ at $z \sim 4$ (see Sect. 3.3.2 for a discussion about $\phi^{*}$ at $z>2$ ). The slope of the LF remains a major source of uncertainty at these redshifts, but also the computation of the dust attenuation. For example, Bouwens et al. (2011) recently revised their previous $A_{\mathrm{FUV}}$ computation (Bouwens et al. 2009): at $z \sim 4$, their dust attenuation correction factor (computed for $L \gtrsim 0$ ) is now $\sim 40 \%$ lower than before. This leads to a total dustcorrected SFRD lower by $\sim 0.2$ dex, giving further support to our result of a low SFRD at this redshift (see also Castellano et al. 2012). Interestingly, the dust attenuation at $z \sim 4$ in Bouwens et al. (2009) was closer to our $A_{\mathrm{FUV}}$, but their new determination of SFRD is closer to our SFRD value.

In the bottom panel of Fig. 5, we add also SFRD data points in the literature, derived using other SFR calibrators than the UV one, mainly at $z<2$. The non FUV-derived SFRD estimates do not increase the scatter observed with the FUV-derived ones. At $z \leq 2$, the cosmic SFRD evolution can be also derived from the stellar mass density assembly, that is from the integrated history of the SFRD down to a given epoch. For instance, we take results from (Ilbert et al. 2010, their Tables 2 and 3), who have computed the stellar mass functions and $\rho_{\text {star }}$ densities over the multi-wavelength COSMOS field (Scoville et al. 2007). From $z=0.2$ to $z=2$, in each redshift bin we obtain $\rho_{\text {star }}$ densities in summing the individual stellar mass densities determined for the "quiescent", "intermediate activity" and "high activity" galaxy populations. Assuming a linear fit, we recover its evolution with redshift, $\rho_{\text {star }}(z)$, and the implied $\operatorname{SFRD}(z)$ with the recipe detailed in Wilkins et al. (2008) ${ }^{6}$. Note that we assume that $30 \%$ of the created stellar mass returned into the interstellar medium: as suggested by Prantzos (2008), this is the average fraction derived using different IMF. As shown in Fig. 5, the resulting inferred $\operatorname{SFRD}(z)$ agrees on average with our direct $\operatorname{SFRD}(\mathrm{z})$ estimate. Nevertheless, there is some discrepancy $(\sim 0.2$ dex $)$ around $z \sim 1$, which happens at the cosmic dust attenuation peak (see Fig. 4) within the star-forming population.

\footnotetext{
5 Note that also uncertainties are often computed in different ways in each galaxy survey, and they are sometimes underestimated because not all-inclusive of many sources of errors. This further increases the nonhomogeneity of the SFRDs determinations.

6 Stellar masses in Ilbert et al. (2010) are computed using a Chabrier (2003) Initial Mass Function, that we convert to a Salpeter (1955) IMF.
} 

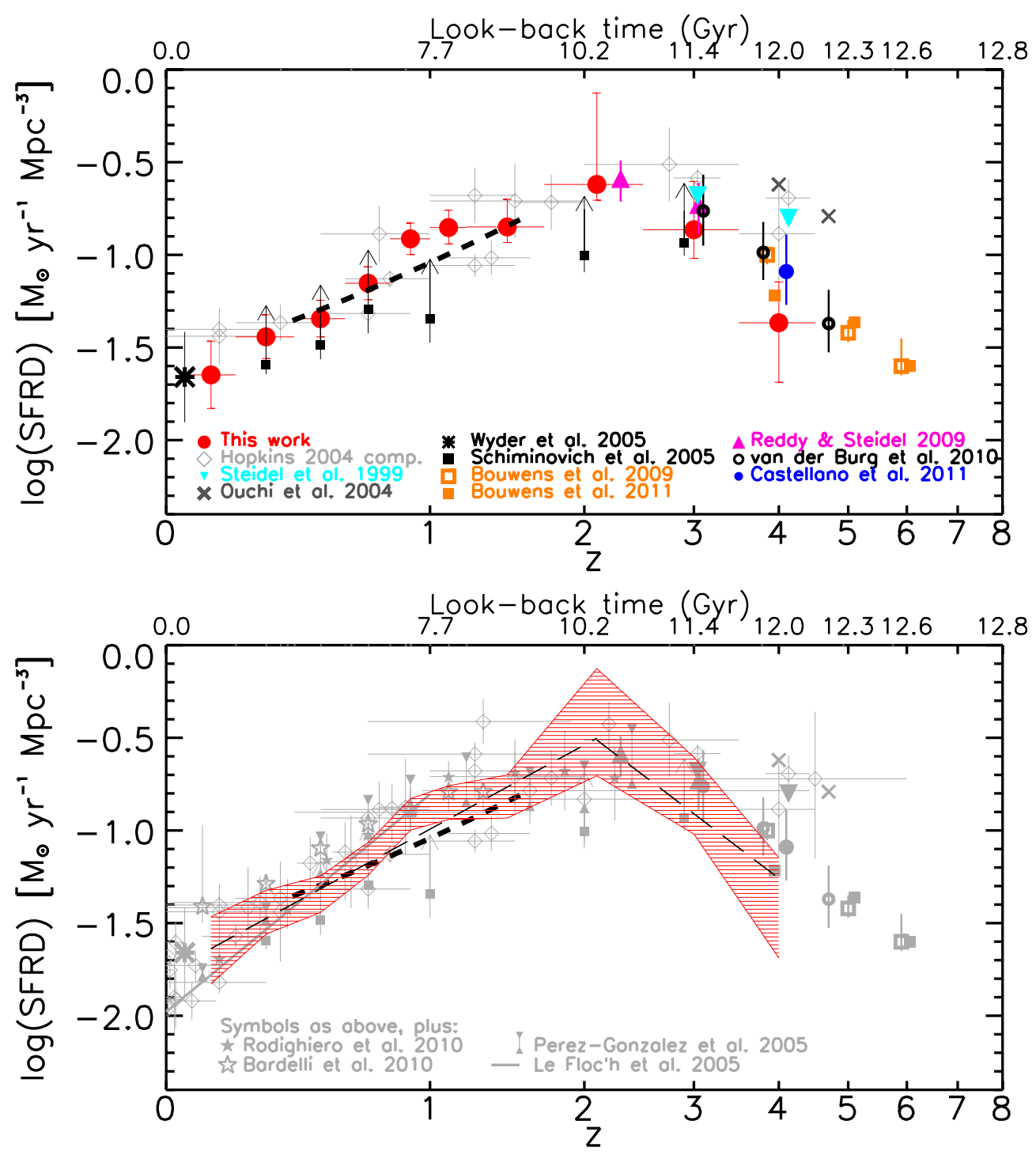

Fig. 5. Top panel. Total dust-corrected UV-derived SFRDs as a function of redshift from the VVDS Deep+Ultra-Deep sample (red filled circles, see Table 1). Our uncertainties are explained in Sect. 3. The black dashed line is the SFRD(z) implied from the stellar mass density in Ilbert et al. (2010) (see text). We overplot other results from the literature, as detailed in the labels and at the end of the caption. Our SFRDs, as well as those from the literature, are derived using the FUV-band LDs converted into SFRD with the scaling relation from Madau et al. (1998). All data have been homogenised with the same IMF (Salpeter 1955). Bottom panel. Total dust-corrected UV-derived SFRDs as a function of redshift from the VVDS Deep+Ultra-Deep sample (red shaded area, corresponding to red circles and error bars in the top panel). The two long-dashed lines represent two fits to our SFRDs in the form $\propto(1+z)^{\beta}$ (see text for details). Gray points are from literature, derived from various SFR calibrators (UV, emission lines, IR, radio): they include the literature points as in the top panel, plus other works as in the labels. List of references, top panel. Compilation in Hopkins (2004) taking only the FUV and NUV determinations (grey open diamonds); Steidel et al. (1999) (LBG sample, cyan solid upside-down triangles); Ouchi et al. (2004) (SDF and SXDF LBGs sample, thick grey crosses); Wyder et al. (2005) (GALEX-2dFGRS, bold asterisk); Schiminovich et al. (2005) (GALEX-VVDS at $z<1.2$ and HDF above, small solid squares with arrows); Bouwens et al. (2009) and Bouwens et al. (2011) (LBGs, orange open and filled squares, respectively); Reddy \& Steidel (2009) (LBG, solid magenta triangles; van der Burg et al. (2010) (CFHTLS, black open circles); Castellano et al. (2012) (LBG, blue open circles). List of references, bottom panel. All the points in the top panel, plus: grey thin diamonds comes from the entire compilation reported in Hopkins (2004); Pérez-González et al. (2005) (12 $\mu$ m, upside-down and normal solid triangles are the lower and upper limits; Rodighiero et al. (2010) (LIR Spitzer VVDS-SWIRE and GOODS, solid stars); Bardelli et al. (2010) (radio VLA-zCOSMOS, open stars); Le Floc'h et al. (2005) (24 $\mu \mathrm{m}$ Spitzer CDFS, solid line; from their Fig. 14).

In summary, the VVDS Deep+Ultra-Deep survey traces continuously the cosmic dust-corrected SFRD over $\sim 12$ Gyr in a comprehensive way, with homogeneous treatment of data, sources of errors, and dust attenuation correction. A clear peak of the SFRD emerges at $z \sim 2$, preceded at earlier cosmic times by a rapid increase by a factor $\sim 6$ within $\sim 2 \mathrm{Gyr}$, and followed by a decrease by a factor $\sim 12$ within $\sim 10 \mathrm{Gyr}$, down to $z \sim 0$. For the first time, the SFRD evolution is genuinely established over 12 Gyr.

\subsection{The contribution of galaxies with different luminosities}

The relative contributions to the total SFRD of distinct galaxy populations vary with redshift, and it is their combination that shapes the global SFRD evolution. Tresse et al. (2007) have assessed the contributions of galaxies with different luminosities to the non dust-corrected FUV-band LDs, showing, for instance, that at $z \sim 3.5$, the different FUV populations equally contribute to the total LD. Then, as the Universe is ageing, the contribution 


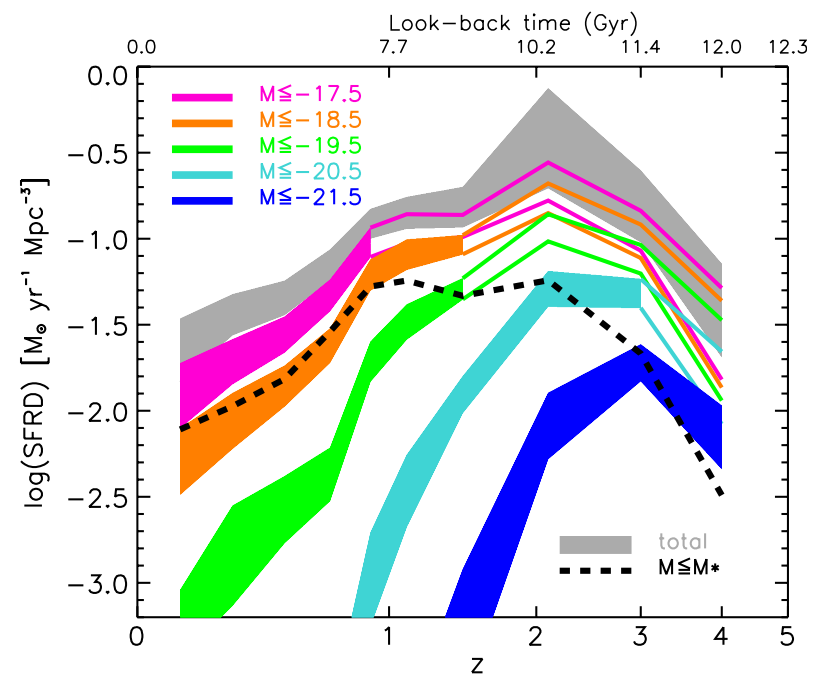

Fig. 6. The dust-corrected SFRD from galaxies with different luminosity limits, as described in the labels. The background gray shaded area is the total SFRD, while the SFRD for different luminosity cuts decreases for brighter galaxies. The luminosity limits are kept fixed at all redshifts. The shaded area for each SFRD accounts for the same kind of errors as for the total SFRD (STY ellipses, cosmic variance, Poisson noise, weighting scheme, and at $z>1.7$ also the span of $\alpha$ between $\alpha=-1.1$ and $\alpha=-1.73$ ). When the luminosity limit is fainter than the LF magnitude bias (see Sect. 3.1) at a given redshift, the area between the lower and upper 1- $\sigma$ error is not filled but left empty. The dashed black line is the SFRD from galaxies brighter than $M^{*}$ at each redshift (i.e., the luminosity cut varies with redshift following $M^{*}$ ). At each redshift, error bars on this SFRD are similar to those for the cut in luminosity closest to $M^{*}$, and we do not overplot them for clarity. We apply to all the points the same dust correction as for the total SFRD, as we do not detect a clear dependence of $E(B-V)$ on FUV luminosity (see discussion in Sect. 5.1).

of the luminous FUV population $\left(M_{\mathrm{FUV}}<-19\right)$ dimes, while the one of the fainter population increases. With our present data, we can directly study the contribution of galaxies with different luminosities to the dust-corrected SFRD. We defer to a future work the analysis on how galaxies contribute to the total SFRD as a function of their stellar mass.

Figure 6 shows the dust-corrected SFRD of galaxies with different luminosity upper limits: $M_{\mathrm{FUV}} \leq-17.5$, $-18.5,-19.5,-20.5,-21.5$ and $M_{\mathrm{FUV}} \leq M_{\mathrm{FUV}}^{*}$ (we remind that $M_{\mathrm{FUV}}^{*}$ varies with $z$, see Table 1 ). We apply to each SFRD the same dust correction derived for the total SFRD, since we do not detect any significant $E(B-V)$ colour dependence with the FUV luminosity (see Sect. 5.1). We see that, as times goes by, the total SFRD is dominated by fainter and fainter galaxies. In fact, bright galaxies with $M_{\mathrm{FUV}} \leq-21.5$ contribute little to the total SFRD for $z \lesssim 2$, and this holds for galaxies with $M_{\mathrm{FUV}} \leq-20.5$ at $z \lesssim 1$ and with $M_{\mathrm{FUV}} \leq-19.5$ at $z \lesssim 0.8$.

To investigate further these trends, we made 6 classes of galaxies within the following $M_{\mathrm{FUV}}$ luminosity ranges: $\geq-17.5$, $[-17.5,-18.5],[-18.5,-19.5],[-19.5,-20.5],[-20.5,-21.5]$ and $\leq-21.5$ (see Fig. 7). We describe below the three main cosmic eras of the SFRD history.

From $z=4$ to $z=2$. During this period, the SFRD from each galaxy subclass increases, with the only exception of the brightest galaxies $\left(M_{\mathrm{FUV}}<-21.5\right)$, whose SFRD starts decreasing at $z \sim 3$. Even including all sources of uncertainties, the contribution of these brightest galaxies to the total SFRD is a maximum of $20 \%$ at $z \sim 3$ and decreases to a maximum of $10 \%$ at $z=2$. The net effect is that the total SFRD increases

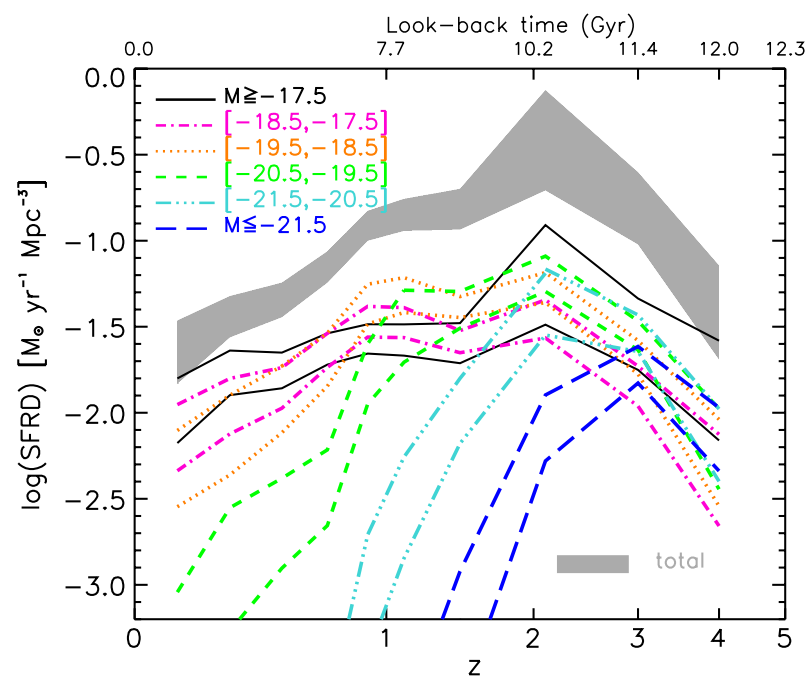

Fig. 7. As in Fig. 6, but this time galaxies are divided in narrow luminosity bins, as indicated in the labels. The first and last bins are actually the extremes of the luminosity distribution (the faintest and the brightest galaxies). Also in this case, as in Fig. 6, the errors include all sources of uncertainty. The two lines for each luminosity bin define the region of $\pm 1-\sigma$ error. Not to crowd the plot, as the plotted quantities have larger error bars than in Fig. 6, here we do not distinguish when the luminosity bin considered is fainter or brighter than the LF bias. This can be inferred by Fig. 6 .

from $z=4$ to $z=2$, because the SFRDs of the main contributors $\left(M_{\mathrm{FUV}}>-21.5\right)$ increase as well.

From $z=2$ to $z=1$. The period corresponds to the change of the shape and normalisation of the FUV LFs (see Figs. 1 and 8 ). Thus, the trends are more differentiated from class to class: the SFRDs for [-19.5,-17.5] stay about constant, for $>-17.5$ and $[-20.5,-19.5]$ mildly decrease, for $<-20.5$ sharply drop. The net effect is however a global decrease, the highest star-forming populations being shut down (galaxies brighter than $M_{\mathrm{FUV}}=-20.5$ contribute maximum by $\left.3 \%\right)$.

From $z=1$ to $z \sim 0$. Through this period, the individual SFRDs reach a general decreasing trend, particularly steep for $[-20.5,-19.5]$ galaxies. As times goes by, the less galaxies are forming stars, the larger is their contribution to the total SFRD. The net effect is that the total SFRD decreases from $z=1$ to $z \sim 0$.

To summarise, the total SFRD evolution (with an increase from $z=4$ to $z=2$, a peak at $z=2$ and a decrease from $z=2$ to $z=0$ ) is driven by different galaxy classes at different epochs. In particular, the percentage contribution to the total SFRD of all galaxy classes is roughly the same at $z=2$, but the peak of the total SFRD at this redshift is mainly due to a similar peak of the SFRD from galaxies with $-21.5 \leq M_{\mathrm{FUV}}<-19.5\left(L \gtrsim L_{z=2}^{*}\right)$. In contrast, the contribution of the most luminous star-forming galaxies reaches its maximum at higher redshift $(z \sim 3)$. We note that the faintest galaxies $\left(M_{\mathrm{FUV}}>-17.5\right)$ are the only ones that show a continuously decreasing SFRD from $z=2$ to $z=0$.

Figure 7 shows that at $z>3$ each FUV luminosity class shows a very similar SFRD trend with $z$, while for $z<3$ the contributions of the various classes to the SFRD become more and more dissimilar as times goes by. This implies i) the presence of a very high- $z$ population not yet strongly affected by star formation regulation/quenching mechanisms and ii) a downsizing pattern in the SFR history.

We remark that the contribution of the faintest galaxies $\left(M_{\mathrm{FUV}}>-17.5\right)$ is clearly affected by the shape (and its 
evolution) of the LF. We refer the reader to Tresse et al. (2007) for an extensive analysis of the role of $\alpha$ in the relative contribution of faint galaxies to the total LD. The difficulty in constraining $\alpha$, and the spread in typical luminosity of the targeted populations, are surely two important sources of uncertainty in the literature in the measurement of the total SFRD shape in this redshift range.

\section{Summary and discussion}

In this work, we have computed the rest-frame FUV luminosity functions, luminosity densities and dust-corrected star formation rate densities within a single and deep redshift survey, merging the VVDS Deep and VVDS Ultra-Deep data sets (with overall $17.5 \leq I_{A B} \leq 24.75$ ), over a 12 Gyr cosmic time baseline $(0.05<z \leq 4.5)$. We also derived the average dust attenuation in the entire redshift range explored. Our data constitute a large improvement with respect to those used in previous VVDS studies: i) the Deep survey includes more redshifts, ii) repeated observations (leading to $100 \%$ secure redshift measurement for previous lower quality spectra) enabled us to correct our $n(z)$ for flags 1 and 2, iii) we use the Ultra-Deep sample, pushing 0.75 mag deeper, iv) we have deeper optical photometry, in particular in the $u^{*}$-band and $z$-band, and $\mathrm{v}$ ) we have used new near-infrared deeper photometry.

Our results are summarised as follows.

- We find a flat and constant faint-end slope in the FUV-band $\mathrm{LF}$ at $z<1.7(\alpha \sim 1)$. We verified that this is unlikely to be the result of missing faint galaxies from our $I$-band selection, and that dust may have a role (see discussion in Sect. 3.3). At the same time, $M_{\mathrm{FUV}}^{*}$ increases by $\sim 1.5 \mathrm{mag}$ from $z \sim 0$ to $z \sim 1.2$, while $\phi^{*}$ starts decreasing at $z>0.7$ (more than a factor of 2 decrease from $z \sim 0.7$ to $z \sim 1.5$ ).

- At $z>1.7$, we set $\alpha$ evolving with $(1+z)$, and this way it becomes as steep as -1.73 at $z \simeq 4$, consistent with values from deep photometric studies. In the meanwhile, $M_{\mathrm{FUV}}^{*}$ keeps on brightening (by an added 2.5 mag up to $z \sim 4$ ), and $\phi^{*}$ decreasing (by another factor of $\sim 40$ up to $z \sim 4$ ). We find that at $z=2,3,4$ our $M_{\mathrm{FUV}}^{*}$ is on average brighter than what has been found in previous works, and that $\phi^{*}$ is on average smaller, in particular at $z \sim 4$. A summary raw scheme of these trends within $0<z<4$ is shown in Fig. 8.

- We find that at $z \sim 3$, while the projected number counts of I-selected galaxies like VVDS are at least twice larger than the projected number counts of LBG-selected galaxies (see Le Fèvre et al., in prep.), the VVDS LF has about 50\% higher density at $M_{\mathrm{FUV}} \sim-22$ than the LF derived from LBG counts. To interpret this apparent discrepancy, we verified the importance of the redshift distribution $n(z)$ shape when transforming number counts (as a function of the observed magnitude) into a LF, or vice versa. Also, when comparing number counts of two different samples in a given redshift range, one has to take into account the $n(z)$ of the two data sets.

- We derived the evolution of the dust-attenuation in the FUVband $\left(A_{\mathrm{FUV}}\right)$ in the range $0.05<z \leq 4.5$, using a SED fitting method, in a consistent way from a single survey with a well controlled selection function. We find a continuous increase of $A_{\mathrm{FUV}}$ by $\sim 1 \mathrm{mag}$ from $z \sim 4.5$ to $z \sim 1$ (with the increase being very slow at $1.5>z>1$ ), then a decrease by the same amount from $z \sim 1$ to $z \sim 0$. This is the first time that the $A_{\text {FUV }}$ evolution has been assessed homogeneously on such a broad redshift range.

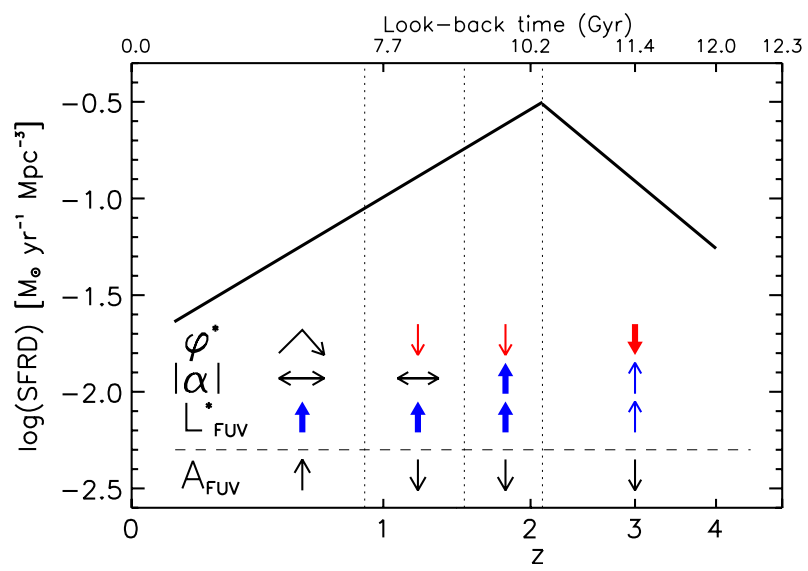

Fig. 8. Scheme of the total dust-corrected SFRD as a function of redshift, as found in this work (solid broken line, see Sect. 5.2). We distinguish four epochs, separated with vertical dotted lines. At each epoch, arrows indicate the behaviour of the three Schechter parameters and $A_{\mathrm{FUV}}$ : arrows pointing up mean that the parameter is increasing with increasing redshift, arrows pointing down mean that the parameter is decreasing. Red arrows indicate trends that in principle should decrease the total SFRD, the opposite is for blue arrows. Thick arrows indicate which parameter is driving the SFRD evolution at that given epoch. In this raw scheme, we neither consider $A_{\mathrm{FUV}}$ dominating, nor making increase or decrease the SFRD: clearly, its trend enhances or weakens the behaviour of the LD when it is transformed to dust-corrected SFRD, but the peak at $z \sim 2$ is already found in the LD.

- We traced the dust-corrected SFRD evolution over the past $\sim 12$ Gyr. It can be schematically fitted as $\operatorname{SFRD}(z) \propto(1+z)^{\beta}$, with $\beta=2.6 \pm 0.4$ at $z<2$ and $\beta=-3.6 \pm 1.9$ at $z>2$ (see Figs. 5 and 8 ). Thanks to the homogeneity of our data over such a cosmic time, we have been able to unveil the presence of a peak at $z \sim 2$ in the cosmic SFR history. This peak is preceded by a rapid increase by a factor 6 from $z \sim 4.5$, then followed by a general decrease by a factor 12 to $z \sim 0$. We remark that the epoch of the peak of the dust-corrected SFRD $(z \sim 2)$ does not coincide with that of the maximum of the dust attenuation evolution $(z \sim 1)$, and that a peak at $z \sim 2$ is already present in the evolution of our LD.

- Studying the contribution to the total SFRD of galaxy populations with different properties, we find that as times goes by, the total SFRD is dominated by fainter and fainter galaxies. Moreover, the presence of a SFRD peak at $z \sim 2$ is mainly due to a similar peak within the population of galaxies with $-21.5 \leq M_{\mathrm{FUV}} \leq-19.5$, while the most extreme star-forming galaxies reaches their maximum activity at higher redshift.

Our data therefore consistently show a peak in the LD and SFRD at $z \sim 2$, where the LF is well constrained. While the increase in SFRD from $z=4$ to $z=2$ is not in question, the exact amplitude of this increase remains to be investigated, as the faint end slope of the LF is still unconstrained even from our very deep spectroscopic survey. Other measurements of the SFRD beyond $z=2$, using e.g. photometric redshifts derived from multi-band imaging (for example Gabasch et al. 2004; Sawicki \& Thompson 2006; Bouwens et al. 2009), go deeper than spectroscopic samples but carry significant associated uncertainties either on the photometric redshifts or because of the small size of the fields and/or samples. It is then fair to say that the exact increase of the SFRD from higher redshift to $z \sim 2$ remains to be robustly quantified. 
The correct determination of the shape of the SFRD evolution is necessary to understand which physical processes mostly affect galaxy evolution. The SFRD is the result of the transformation of gas into stars and therefore requires a significant gas reservoir to sustain a strong star formation rate for a long time. A number of processes are expected to modify the gas reservoir hence the SFR, including the efficiency of star formation, cold accretion along the cosmic web filaments, mergers with gas-rich galaxies, stellar feedback, SN feedback blowing gas out from the galaxy core, AGN feedback, cosmic photoionizing radiation, or environment effects which may result in star formation quenching (e.g., among many others, White \& Frenk 1991; Efstathiou 1992; Cole et al. 1994, 2000; Di Matteo et al. 2005; Baugh 2006; Cox et al. 2008; Dekel et al. 2009; de Ravel et al. 2009). The exact balance of these different processes along cosmic time will result in the observed SFRD. The SFRD peak that we find at $z \simeq 2$ is produced by galaxies with $L$ close to or even brighter than the $L^{*}$ of that redshift, requiring that significant gas reservoirs still exist at this epoch and are probably replenished by cold accretion and wet mergers, while feedback or quenching processes are not yet strong enough to lower the SF. The knee of the rest-frame FUV LF shape is smoothed away at $z \gtrsim 2$, i.e. there is not anymore a clear distinction between the high and intermediate star-forming galaxy densities. It does not mean that the high-z gas-rich galaxies form a homogeneous population, but likely that the long time-scale ( $t>3 \mathrm{Gyr}$ ) processes do not heavily affect the galaxy SFR yet. Knowing the SFRD, we may hope to identify the relative contribution of these different processes at different epochs.

Using simulations (semi-analytical galaxy evolution models, smoothed particle hydrodynamics simulation), several authors have attempted to reproduce the observed Cosmic Star Formation History with theoretical predictions (e.g. Baugh et al. 2005; Somerville et al. 2008; Fontanot et al. 2009; Hopkins et al. 2010; van de Voort et al. 2011). As a recent example, Weinmann et al. (2011) use semi-analytical galaxy evolution models to predict the SFRD evolution (see their Fig. 10). They start from a standard model of galaxy evolution, and produce slightly different versions of it by tuning one or more ingredients at a time (such as star formation efficiency, stellar feedback, merger processes...). Although the main goal of their work is not to reproduce the SFRD evolution, from their Fig. 10 it is clear that models in which different physical processes have been enhanced or depressed predict significantly different Cosmic Star Formation Histories. All of their models reproduce qualitatively the fast SFRD increase from $z \sim 0$ to $z \sim 1$, but at $z>1$ the predicted SFRDs have different behaviours, showing growths and decreases at different rates, in some cases a plateau, in some cases a more or less pronounced peak at different possible redshifts. It is worth noticing that one of the models (where feedback has been tuned) predicts a sharp peak in the SFRD evolution at $z \sim 1.5$, qualitatively similar to the one that we find at $z \sim 2$. It is not the aim of this paper to compare in details our findings with model predictions, and we will address this issue in more details in a future work. Here we want only to stress the importance of bringing strong observational constraints on the SFRD from a unique and homogeneous galaxy sample covering a large cosmic time of $\sim 12$ Gyr, which will need to be reproduced by next generation models.

Another remarkable finding of our work is that the peak of the dust and the SFR do not coincide, differently from what one could have naively thought in the case the dust is immediately released into the ISM a short time after supernova explosions of massive, short-lived stars which dominate the SFR.
These two peaks are separated by $\sim 2.5 \mathrm{Gyr}$, which is a long period if one considers that the dust production rate peak is below 1 Gyr for SNII, but it is at 3-4 Gyr for intermediate-mass stars (see Dwek 1998). Nevertheless the dust reaches a sort of plateau from $z \sim 1.5$ (with a maximum at $z \sim 1$ ), i.e. $1 \mathrm{Gyr}$ after the peak of the SFRD. Recently, Fukugita (2011) suggested that the dust must survive on much longer time scales than what has been previously thought and that half of the dust could be produced by SNII and the other half by intermediate-mass (1-8 solar masses), long-lived stars. If we assume that the SNII dust production peaks very shortly after the SFRD peak, then the dust peak that we observe at $z \sim 1$ is likely due to intermediate-mass, long-lived stars producing their peak of dust on a delayed time. In particular, the AGB stars release dust through intense mass loss, and most efficiently at the very end stages of evolution (Gall et al. 2011). The peak of attenuation is higher than SNII simply because these stars are much more numerous than very massive, short-lived OB stars, assuming a universal IMF. It would explain also that at $z>2$, the low level of dust attenuation is mainly due to dust produced by SNII, while at $z<2$, it is resulting from the combination of dust from SNII and intermediate-mass stars. Our findings, combined with the above-mentioned times scales, may imply that dust is not only quickly formed (thanks to SNII ejecta and remnants), but also quickly destroyed, because the peak of attenuation is only $\sim 2.5$ Gyr after the SFRD peak, a shorter time-scale than the typical one for dust production by intermediate-mass stars (3-4 Gyr). Surely, like in the case of the SFRD evolution, our findings about the general evolution of the FUV-band dust attenuation in such a broad cosmic epoch ( $12 \mathrm{Gyr})$ will constitute an important reference for future models.

Acknowledgements. We would like to thank the referee for useful suggestions and comments, that improved the manuscript. We thank S. Boissier for useful discussions. O.C. thanks V. Buat, D. Burgarella and L. Cortese for interesting conversations. This work has been partially supported by the CNRS-INSU and its Programme National Cosmologie-Galaxies (France) and by INAF grant COFIN 2010.

\section{References}

Arnouts, S., Schiminovich, D., Ilbert, O., et al. 2005, ApJ, 619, L43 Bardelli, S., Schinnerer, E., Smolčic, V., et al. 2010, A\&A, 511, A1 Baugh, C. M. 2006, Rep. Prog. Phys., 69, 3101

Baugh, C. M., Lacey, C. G., Frenk, C. S., et al. 2005, MNRAS, 356, 1191

Bielby, R., Hudelot, P., McCracken, H. J., et al. 2011, A\&A, submitted [arXiv: 1111.6997]

Bouwens, R. J., Illingworth, G. D., Franx, M., \& Ford, H. 2007, ApJ, 670, 928

Bouwens, R. J., Illingworth, G. D., Franx, M., et al. 2009, ApJ, 705, 936

Bouwens, R. J., Illingworth, G. D., Oesch, P. A., et al. 2011, ApJ, submitted [arXiv: 1109.0994]

Bruzual, G., \& Charlot, S. 2003, MNRAS, 344, 1000

Calzetti, D. 2008, in Pathways Through an Eclectic Universe, ed. J. H. Knapen, T. J. Mahoney, \& A. Vazdekis, ASP Conf. Ser., 390, 121

Calzetti, D., Armus, L., Bohlin, R. C., et al. 2000, ApJ, 533, 682

Cassata, P., Le Fèvre, O., Garilli, B., et al. 2011, A\&A, 525, A143

Castellano, M., Fontana, A., Grazian, A., et al. 2012, A\&A, in press DOI: $10.1051 / 0004-6361 / 201118050$

Chabrier, G. 2003, PASP, 115, 763

Cole, S., Aragon-Salamanca, A., Frenk, C. S., Navarro, J. F., \& Zepf, S. E. 1994, MNRAS, 271, 781

Cole, S., Lacey, C. G., Baugh, C. M., \& Frenk, C. S. 2000, MNRAS, 319, 168

Connolly, A. J., Szalay, A. S., Dickinson, M., Subbarao, M. U., \& Brunner, R. J. 1997, ApJ, 486, L11

Cortese, L., Boselli, A., Buat, V., et al. 2006, ApJ, 637, 242

Cowie, L. L., Songaila, A., \& Barger, A. J. 1999, AJ, 118, 603

Cox, T. J., Jonsson, P., Somerville, R. S., Primack, J. R., \& Dekel, A. 2008, MNRAS, 384, 386

de Ravel, L., Le Fèvre, O., Tresse, L., et al. 2009, A\&A, 498, 379

Dekel, A., Birnboim, Y., Engel, G., et al. 2009, Nature, 457, 451 
Di Matteo, T., Springel, V., \& Hernquist, L. 2005, Nature, 433, 604 Driver, S. P., \& Robotham, A. S. G. 2010, MNRAS, 407, 2131 Driver, S. P., Popescu, C. C., Tuffs, R. J., et al. 2008, ApJ, 678, L101

Dwek, E. 1998, ApJ, 501, 643

Efstathiou, G. 1992, MNRAS, 256, 43P

Fioc, M., \& Rocca-Volmerange, B. 1997, A\&A, 326, 950

Fontanot, F., De Lucia, G., Monaco, P., Somerville, R. S., \& Santini, P. 2009, MNRAS, 397, 1776

Fukugita, M. 2011, MNRAS, submitted [arXiv: 1103.4191]

Gabasch, A., Bender, R., Seitz, S., et al. 2004, A\&A, 421, 41

Gall, C., Hjorth, J., \& Andersen, A. C. 2011, A\&AR, 19, 43

Garilli, B., Le Fèvre, O., Guzzo, L., et al. 2008, A\&A, 486, 683

Hopkins, A. M. 2004, ApJ, 615, 209

Hopkins, A. M., \& Beacom, J. F. 2006, ApJ, 651, 142

Hopkins, P. F., Younger, J. D., Hayward, C. C., Narayanan, D., \& Hernquist, L. 2010, MNRAS, 402, 1693

Ilbert, O., Tresse, L., Arnouts, S., et al. 2004, MNRAS, 351, 541

Ilbert, O., Tresse, L., Zucca, E., et al. 2005, A\&A, 439, 863

Ilbert, O., Arnouts, S., McCracken, H. J., et al. 2006, A\&A, 457, 841

Ilbert, O., Capak, P., Salvato, M., et al. 2009, ApJ, 690, 1236

Ilbert, O., Salvato, M., Le Floc'h, E., et al. 2010, ApJ, 709, 644

Iwata, I., Ohta, K., Tamura, N., et al. 2007, MNRAS, 376, 1557

Kennicutt, Jr., R. C. 1998, ARA\&A, 36, 189

Kong, X., Charlot, S., Brinchmann, J., \& Fall, S. M. 2004, MNRAS, 349, 769

Le Fèvre, O., Saisse, M., Mancini, D., et al. 2003, in Instrument Design and Performance for Optical/Infrared Ground-based Telescopes, ed. M. Iye, \& A. F. M. Moorwood, Proc. SPIE, 4841, 1670

Le Fèvre, O., Mellier, Y, McCracken, H. J., et al, 2004a, A\&A, 417, 839

Le Fèvre, O., Vettolani, G., Paltani, S., et al. 2004b, A\&A, 428, 1043

Le Fèvre, O., Paltani, S., Arnouts, S., et al. 2005a, Nature, 437, 519

Le Fèvre, O., Vettolani, G., Garilli, B., et al. 2005b, A\&A, 439, 845

Le Floc'h, E., Papovich, C., Dole, H., et al. 2005, ApJ, 632, 169

Lilly, S. J., Le Fevre, O., Hammer, F., \& Crampton, D. 1996, ApJ, 460, L1

Madau, P., Ferguson, H. C., Dickinson, M. E., et al. 1996, MNRAS, 283, 1388

Madau, P., Pozzetti, L., \& Dickinson, M. 1998, ApJ, 498, 106

McCracken, H. J., Radovich, M., Bertin, E., et al. 2003, A\&A, 410, 17

Meurer, G. R., Heckman, T. M., \& Calzetti, D. 1999, ApJ, 521, 64

Oesch, P. A., Bouwens, R. J., Carollo, C. M., et al. 2010, ApJ, 725, L150

Ouchi, M., Shimasaku, K., Okamura, S., et al. 2004, ApJ, 611, 660

Paltani, S., Le Fèvre, O., Ilbert, O., et al. 2007, A\&A, 463, 873

Pérez-González, P. G., Rieke, G. H., Egami, E., et al. 2005, ApJ, 630, 82

Polletta, M., Tajer, M., Maraschi, L., et al. 2007, ApJ, 663, 81

Prantzos, N. 2008, in EAS Publ. Ser. 32, ed. C. Charbonnel, \& J.-P. Zahn, 311

Reddy, N. A., \& Steidel, C. C. 2009, ApJ, 692, 778

Rodighiero, G., Vaccari, M., Franceschini, A., et al. 2010, A\&A, 515, A8

Ryan, Jr., R. E., Hathi, N. P., Cohen, S. H., et al. 2007, ApJ, 668, 839

Salpeter, E. E. 1955, ApJ, 121, 161

Sawicki, M., \& Thompson, D. 2006, ApJ, 642, 653
Schechter, P. 1976, ApJ, 203, 297

Schiminovich, D., Ilbert, O., Arnouts, S., et al. 2005, ApJ, 619, L47

Scoville, N., Aussel, H., Brusa, M., et al. 2007, ApJS, 172, 1

Somerville, R. S., Lee, K., Ferguson, H. C., et al. 2004, ApJ, 600, L171

Somerville, R. S., Hopkins, P. F., Cox, T. J., Robertson, B. E., \& Hernquist, L. 2008, MNRAS, 391, 481

Steidel, C. C., Adelberger, K. L., Giavalisco, M., Dickinson, M., \& Pettini, M. 1999, ApJ, 519, 1

Sullivan, M., Treyer, M. A., Ellis, R. S., et al. 2000, MNRAS, 312, 442

Takeuchi, T. T., Buat, V., \& Burgarella, D. 2005, A\&A, 440, L17

Trenti, M., \& Stiavelli, M. 2008, ApJ, 676, 767

Tresse, L., \& Maddox, S. J. 1998, ApJ, 495, 691

Tresse, L., Maddox, S. J., Le Fèvre, O., \& Cuby, J.-G. 2002, MNRAS, 337, 369

Tresse, L., Ilbert, O., Zucca, E., et al. 2007, A\&A, 472, 403

Treyer, M. A., Ellis, R. S., Milliard, B., Donas, J., \& Bridges, T. J. 1998, MNRAS, 300, 303

Treyer, M., Schiminovich, D., Johnson, B., et al. 2007, ApJS, 173, 256

van de Voort, F., Schaye, J., Booth, C. M., \& Dalla Vecchia, C. 2011, MNRAS, 415, 2782

van der Burg, R. F. J., Hildebrandt, H., \& Erben, T. 2010, A\&A, 523, A74

Weinmann, S. M., Neistein, E., \& Dekel, A. 2011, MNRAS, 417, 2737

White, S. D. M., \& Frenk, C. S. 1991, ApJ, 379, 52

Wijesinghe, D. B., da Cunha, E., Hopkins, A. M., et al. 2011, MNRAS, 415, 1002

Wilkins, S. M., Trentham, N., \& Hopkins, A. M. 2008, MNRAS, 385, 687

Wilson, G., Cowie, L. L., Barger, A. J., \& Burke, D. J. 2002, AJ, 124, 1258

Wyder, T. K., Treyer, M. A., Milliard, B., et al. 2005, ApJ, 619, L15

Zucca, E., Ilbert, O., Bardelli, S., et al. 2006, A\&A, 455, 879

1 Laboratoire d'Astrophysique de Marseille (UMR 6110), CNRSUniversité de Provence, 38 rue Frédéric Joliot-Curie, 13388 Marseille Cedex 13, France

2 INAF - Osservatorio Astronomico di Trieste, via Tiepolo 11, 34143 Trieste, Italy e-mail: cucciati@oats.inaf.it

3 INAF - IASF, via Bassini 15, 20133 Milano, Italy

4 Department of Astronomy, University of Massachusetts, Amherst, MA 01003, USA

5 INAF - Osservatorio Astronomico di Bologna, via Ranzani 1, 40127 Bologna, Italy

${ }^{6}$ Department of Physics, Durham University, South Road, Durham DH1 3LE, UK

7 Institut d'Astrophysique de Paris (UMR 7095), CNRS-Université Pierre et Marie Curie, 98bis boulevard Arago, 75014 Paris, France

8 IRA-INAF, via Gobetti 101, 40129 Bologna, Italy

Pages 17 to 23 are available in the electronic edition of the journal at http://www . aanda. org 


\section{Appendix A: The weighting schemes}

We refer the reader to Le Fèvre et al. (2005b) and Le Fèvre et al. (in prep.) for details on the quality flags assigned to redshift measurements in the VVDS Deep and Ultra-Deep surveys, respectively. We detail below the weighting schemes to be applied to our statistical analyses. They account for the selection function of the photometric sources targeted to acquire their spectrum (the Target Sampling Rate, TSR), and for the success to measure a reliable redshift from the spectrum (the Spectroscopic Success Rate, SSR). They are derived for the Deep survey, the UltraDeep survey, and for the merged Deep+Ultra-Deep sample.

To derive the weighting schemes, we made use of the photometric redshifts, zphot, computed as described in Ilbert et al. (2006), but using the more recent T0005 release of CFHTLS photometric data $\left(u^{*}, g^{\prime}, r^{\prime}, i^{\prime}, z^{\prime}\right)$ and the latest near-infrared photometric data available from $\operatorname{WIRDS}\left(J, H\right.$ and $K_{\mathrm{s}}$, Bielby et al. 2011).

We emphasise that we correct the Deep and Ultra-Deep distributions of photometric redshifts, $n$ (zphot), for their failure rate computing the ratio between the spectroscopic redshifts measured with a $>97 \%$ confidence level (flags 3,4$), n\left(z_{f=3,4}\right)$ and their corresponding photometric redshifts, $n\left(\right.$ zphot $\left._{f=3,4}\right)$.

\section{A.1. Weights for the Deep survey}

The Deep survey $\mathrm{TSR}_{\mathrm{d}}$ is defined as $N_{\text {target }}^{\mathrm{d}} / N_{\text {phot }}^{\mathrm{d}}$, where $N_{\text {phot }}^{\mathrm{d}}$ is the number of photometric sources in the Deep survey with $17.5 \leq I_{A B} \leq 24.0$, over $\sim 2200 \operatorname{arcmin}^{2}$ of sky area, and $N_{\text {target }}^{\mathrm{d}}$ is the number of photometric sources targeted for spectroscopic observations. The $\mathrm{TSR}_{\mathrm{d}}$ depends on the projection of the angular size of each object on the $x$-axis of the image (" $x$-radius", see also Ilbert et al. 2005). The weight associated to $\mathrm{TSR}_{\mathrm{d}}$ is $w_{\mathrm{TSR}}^{\mathrm{d}}(r)=1 / T S R_{\mathrm{d}}(r)$, where $r$ is the $x$-radius defined above.

The Deep survey $\mathrm{SSR}_{\mathrm{d}}$ is defined as $N_{\text {spec }}^{\mathrm{d}} / N_{\text {target }}^{\mathrm{d}}$, where $N_{\text {spec }}^{\mathrm{d}}$ is the number of targets with a reliable spectroscopic redshift measurement in the Deep survey. The $\mathrm{SSR}_{\mathrm{d}}$ is a function of both selection magnitude and redshift (see Ilbert et al. 2005). As described in Le Fèvre et al. (in prep.), a fraction of low confidence level spectroscopic redshifts (flags $0,1,2$ ) at $z \geq 1.4$ from the Deep survey have been observed again, leading to a $100 \%$ confidence level in the redshift measurement. Comparing the old redshift distribution $n\left(z_{f=1,2}^{\text {reobserved }}\right)$ to the new one for this subsample, we have remodulated the full $n\left(z_{f=1,2}\right)$ partially using the $n$ (zphot) for $z<1.4$ sources. We have also remodulated the single emission-line redshifts (flags 9$) n\left(z_{f=9}\right)$ according to its $n\left(\right.$ zphot $\left._{f=9}\right)$. This gives:

$$
\begin{aligned}
N_{\mathrm{spec}}^{\mathrm{d}}(m, z)= & N_{f=1,2}(m, z) \times n\left(m, z_{f=1,2}^{\text {reosserved }}\right) / n\left(m, z_{f=1,2}\right) \\
& +N_{f=9}(m, z) \times n\left(m, \text { phot }_{f=9}\right) / n\left(m, z_{f=9}\right) \\
& +N_{f=3,4}(m, z) .
\end{aligned}
$$

The weight associated to $\operatorname{SSR}_{\mathrm{d}}$ is $w_{\mathrm{SSR}}^{\mathrm{d}}(m, z)=1 / \operatorname{SSR} R_{\mathrm{d}}(m, z)$.

In summary, for the Deep survey, we have $N_{\text {spec }}^{\mathrm{d}} \subset N_{\text {target }}^{\mathrm{d}} \subset$ $N_{\text {phot }}^{\mathrm{d}}$ and we apply the weight $w_{\mathrm{d}}(r, m, z)=w_{\mathrm{TSR}}^{\mathrm{d}}(r) w_{\mathrm{SSR}}^{\mathrm{d}}(m, z)$ to each galaxy in $N_{\text {spec }}^{\mathrm{d}}$.

\section{A.2. Weights for the Ultra-Deep survey}

The Ultra-Deep survey covers a $576 \mathrm{arcmin}^{2}$ sky area embedded in the Deep survey area. It is purely flux limited at $23.00 \leq$ $i_{A B}^{\prime} \leq 24.75$ with $N_{\text {phot }}^{\text {ud }}$ photometric sources. Several spectroscopic sources with $23 \leq I_{A B} \leq 24$ had already been observed in the VVDS Deep survey, when we started the Ultra-Deep observations. By excluding them, we obtained a reduced catalogue with $N_{\text {phot }}^{\text {parent }}$ sources available for the Ultra-Deep spectroscopic target selection. The Ultra-Deep Photometric Sampling Rate $\left(\mathrm{PSR}_{\mathrm{ud}}\right)$ is defined as $N_{\text {phot }}^{\mathrm{par} t} / N_{\text {phot }}^{\mathrm{ud}}$. The $\mathrm{PSR}_{\mathrm{ud}}$ depends on the $I$-band apparent magnitude. The weight associated to $\mathrm{PSR}_{\mathrm{ud}}$ is $w_{\mathrm{PSR}}^{\mathrm{ud}}(m)=1 / P S R_{\mathrm{ud}}(m)$.

The Ultra-Deep survey $\mathrm{TSR}_{\mathrm{ud}}$ is defined as $N_{\text {target }}^{\mathrm{ud}} / N_{\text {phot }}^{\mathrm{parent}}$, where $N_{\text {target }}^{\text {ud }}$ is the number of photometric sources targeted for Ultra-Deep spectroscopic observations. The TSR $\mathrm{Td}_{\mathrm{ud}}$ does not depend on any parameter, it is a constant value $(6.5 \%)$. The weight associated to $\mathrm{TSR}_{\mathrm{ud}}$ is $w_{\mathrm{TSR}}^{\mathrm{ud}}=1 / T S R_{\mathrm{ud}}$.

The Ultra-Deep $\operatorname{SSR}_{\mathrm{ud}}$ is defined as $N_{\mathrm{spec}}^{\mathrm{ud}} / N_{\text {target }}^{\mathrm{ud}}$, where $N_{\text {spec }}^{\text {ud }}$ is the number of targets with a reliable spectroscopic redshift measurement in the Ultra-Deep survey. Here $N_{\text {spec }}=$ $N_{f=1.5,2,3,4,9}$, where flags 1.5 is a flag 1 with a photometric redshift in agreement with the spectroscopic redshift (Le Fèvre et al., in prep.). The $\mathrm{SSR}_{\mathrm{ud}}$ is a function of both selection magnitude and redshift. The associated weight is $w_{\mathrm{SSR}}^{\mathrm{ud}}(m, z)=$ $1 / S S R_{\mathrm{ud}}(m, z)$.

In summary, for the Ultra-Deep survey, we have $N_{\text {spec }}^{\text {ud }} \subset$ $N_{\text {target }}^{\text {ud }} \subset N_{\text {phot }}^{\text {parent }} \subset N_{\text {phot }}^{\text {ud }}$ and we apply the weight $w_{\text {ud }}(m, z)=$ $w_{\mathrm{PSR}}^{\mathrm{ud}}(m) w_{\mathrm{TSR}}^{\mathrm{ud}} w_{\mathrm{SSR}}^{\mathrm{ud}}(m, z)$ to each galaxy in $N_{\mathrm{spec}}^{\mathrm{ud}}$.

\section{A.3. Weights for the combined Deep and Ultra-Deep surveys}

We combine the two surveys and we derive an adapted weighting scheme to take advantage of both (i) the large magnitude range covered by the Deep survey $\left(17.5 \leq I_{A B} \leq 24.0\right)$ over a large sky area, and (ii) the depth reached by the Ultra-Deep survey (0.75 mag deeper than the Deep) over a smaller embedded sky area.

As a first step, we need to merge the two catalogues of galaxies in accounting for their different depth, especially for their different LF bias limit (see Sect. 3.1). Since the latter depends on the studied redshift bin, we merge the catalogues in each redshift bin in which we explore the LF, as follows. For the Ultra-Deep survey, we compute the LF bias limits with the method described in Sect. 3.1. For the Deep survey, we adopted more conservative limits with respect to those obtained with the above mentioned method, because of the magnitude range $23 \leq I_{A B} \leq 24$ common with the Ultra-Deep survey. In the Deep and Ultra-Deep samples we keep only galaxies brighter than the respective bias limit, and we merge the two sub-samples into a single catalogue. From now on, the merged catalogue is considered with flux limits within $17.50 \leq I_{A B} \leq 24.75$, over the Deep survey effective sky area.

As a second step, we need to slightly modify the TSR weighting scheme for the merged catalogue since the UltraDeep area is embedded in the Deep area. The Deep+Ultra-Deep $\mathrm{TSR}_{\mathrm{d}+\text { ud }}$ is $\left(N_{\text {target }}^{\mathrm{d}}+N_{\text {target }}^{\text {ud }}\right) / N_{\text {phot }}^{\text {full }}$, where $N_{\text {phot }}^{\text {full }}$ is the full photometric catalogue at $17.50 \leq I_{A B} \leq 24.75$ over $2200 \mathrm{arcmin}^{2}$. The $\mathrm{TSR}_{\mathrm{u}+\mathrm{ud}}$ depends on the $I$-band apparent magnitude (see Fig. A.1). At $17.5 \leq I_{A B}<23.0$, it corresponds to $\mathrm{TSR}_{\mathrm{d}}$ when we compute it as a function of magnitude instead of angular size, while at $24.00<I_{A B} \leq 24.75$, it corresponds to the constant $\mathrm{TSR}_{\mathrm{ud}}$. At $23 \leq I_{A B} \leq 24$, it corresponds to the combination of both TSRs, i.e. at the numerator we have all targets of both the Deep and Ultra-Deep surveys. The weight associated to $\mathrm{TSR}_{\mathrm{d}+\mathrm{ud}}$ is $w_{\mathrm{TSR}}^{\mathrm{d}+\mathrm{ud}}(m)=1 / T S R_{\mathrm{d}+\mathrm{ud}}(m)$. We applied this weight according to the $I$-band apparent magnitude to all Deep survey galaxies 


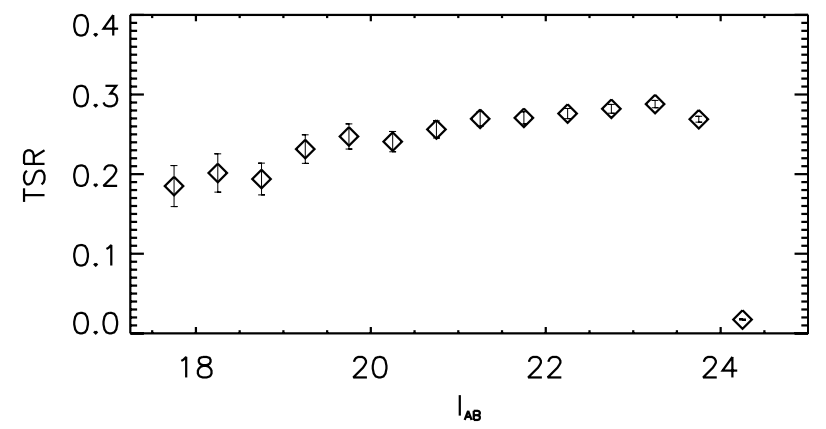

Fig. A.1. Target Sampling Rate (TSR) for the merged Deep+Ultra-Deep catalogue as a function of the $I$-band apparent magnitude. Given the different flux limit of the two surveys (see text), this TSR is obtained using Deep data at $I_{A B}<23$, both Deep and Ultra-Deep data at $23 \leq$ $I_{A B} \leq 24$, and Ultra-Deep data at $I_{A B}>24$. The last point is low because the Ultra-Deep survey TSR is much lower than the Deep survey TSR.

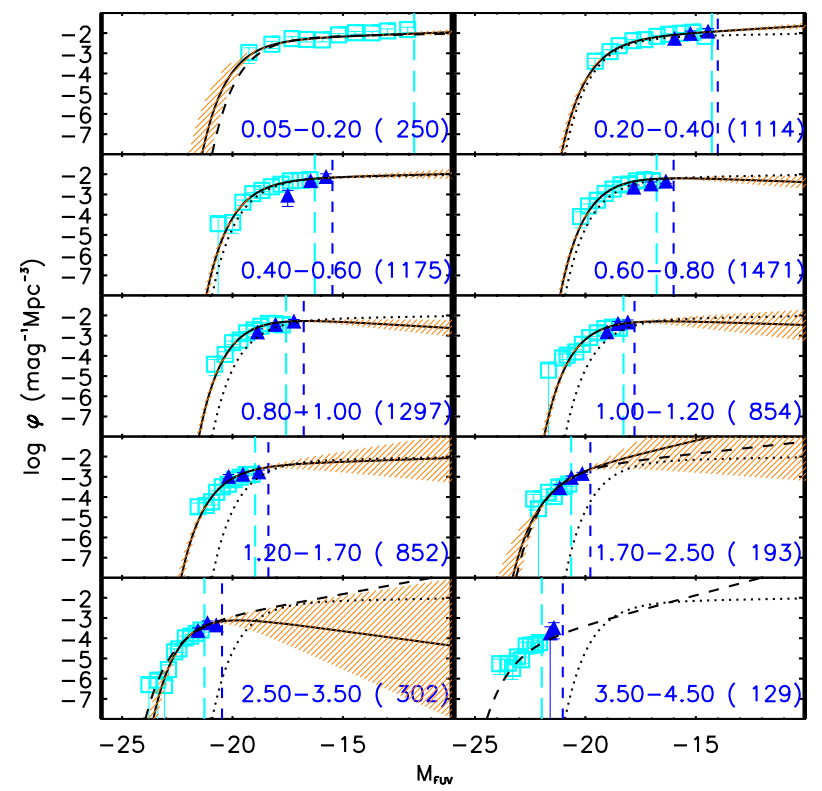

Fig. A.2. Rest-frame FUV $1 / V_{\max }$ LF data points for the Deep survey (cyan empty squares) and the Ultra-Deep survey (blue filled triangles), from $z=0.05$ to $z=4.50$. There is no Ultra-Deep data in the lowest redshift bin. In each panel, the FUV absolute magnitude LF bias limit (see Sect. 3.1) is shown as a vertical cyan long-dashed line for the Deep survey and as a blue short-dashed line for the Ultra-Deep survey. The number quoted in parenthesis is the total number of used galaxies. We overplot the STY determinations of the merged Deep+Ultra-Deep sample, as illustrated in Fig. 1 and tabulated in Table B.1): (i) with free Schechter parameters (solid lines) and its associated error (shaded area), (ii) in setting the faint-end slope at $z>1.7$ (dashed lines), and (iii) in setting $M_{\mathrm{FUV}}^{*}$ at $z<0.2$ (dashed line) and reported as a reference in the $z>0.2$ panels (dotted lines).

and to Ultra-Deep galaxies which are brighter than the LF bias limits of the Deep survey. To the Ultra-Deep galaxies which are fainter than the Deep LF bias limits we applied the $w_{\text {TSR }}^{\mathrm{u}+\mathrm{ud}}$ shown for $I_{A B} \geq 24$.

In summary, for the merged Deep+Ultra-Deep catalogue, we apply the weight $w_{\mathrm{d}}^{\text {merged }}(m, z)=w_{\mathrm{TSR}}^{\mathrm{d}+\mathrm{ud}}(m) w_{\mathrm{SSR}}^{\mathrm{d}}(m, z)$ to $N_{\mathrm{spec}}^{\mathrm{d}}$ and the weight $w_{\mathrm{ud}}^{\text {merged }}(m, z)=w_{\mathrm{TSR}}^{\mathrm{d}+\mathrm{ud}}(m) w_{\mathrm{SSR}}^{\mathrm{ud}}(m, z)$ to $N_{\mathrm{spec}}^{\mathrm{ud}}$.

\section{A.4. The FUV-band LFs using the different weighting schemes}

To verify the robustness of our weights, we separately derive the rest-frame FUV LF of the Deep and the Ultra-Deep surveys, using their independent weighting schemes, as described in Appendices A.1 and A.2. Figure A.2 shows the $1 / V_{\max }$ data points up to the respective LF bias limit (see Sect. 3.1) for the two surveys, together with the STY LFs of the combined Deep and Ultra-Deep surveys (see Sect. 3.2 and Fig. 1), using the merged weighting scheme, as described in Appendix A.3. On one side, the LF data points of the two surveys fully agree from $z=0.2$ to $z=3.5$ in their common range of luminosities. This is an a-posteriori check of the reliability of our individual weighting schemes. On the other side, the STY LFs estimated with the merged sample perfectly overlap the $1 / V_{\max }$ data points estimated with the two individual surveys. This confirms the reliability of the weighting scheme applied to our merged sample of individual surveys with different depth.

\section{Appendix B: Verifying the robustness of our rest-frame FUV-band LF}

In Sect. 3, we have presented our VVDS rest-frame FUV-band LF estimates from $z=0.05$ up to $z=4.5$ (see Fig. 1), and we have tabulated the corresponding best Schechter parameters in Table 1. Figure 2 summarises in one panel our best LF fits. In this Appendix, we give further details and tests we have performed to ensure the robustness of our best rest-frame FUV LF, in particular the flatness of the LF faint-end slope at $z \lesssim 1$ and the low normalisation of the LF at $z \gtrsim 3$. Table B.1 gives our extensive computations of the Schechter parameters with the STY method, leaving the three Schechter parameters totally free. Obviously, we cannot constrain $M^{*}$ at $z \leq 0.2$ nor $\alpha$ at $z>1.7$. We fixed these two parameters as described in Sect. 3.2, and we also report the results in Table B.1. The entries labelled with a black dot are our final choices taken for our studies and also reported in Table 1.

As shown in Fig. 2, our FUV faint-end slope is quite flat $(\alpha \simeq-1)$ at $z<1.7$, that is up to the highest redshift where we can constrain it. The compilation of the $\alpha$ values found in the literature (see Table B.2) shows very scattered values at $0 \lesssim z \lesssim$ 6 . Nevertheless, it is a notable point that $\alpha$ has been generally found steeper (when estimated and not fixed) than our value. To strengthen the validity of our results against possible biases, we report below our LFs computed using photometric redshifts, using only starburst galaxies, and computed in the rest-frame NUV-band.

\section{B.1. The rest-frame FUV LF with zphot}

We derive the VVDS rest-frame FUV $1 / V_{\max }$ estimates using very deep photometric redshifts (zphot, see Appendix A for details). The zphot catalogue is complete up to $I_{A B}=26$ and it consists in $\sim 43000$ photometric sources over the VVDS Deep area. In this case, the weighting scheme is obviously not used. The bias limit has been computed in each $z$ bin as explained in Sect. 3.1, and at any $z$ it is clearly fainter than the bias limit in the VVDS Deep+Ultra-Deep spectroscopic sample.

Results are shown in Fig. B.1, and globally overlap our best rest-frame FUV-band LF determinations using the VVDS Deep+Ultra-Deep spectroscopic sample up to $I_{A B}=24.75$. Thus, our spectroscopic sample (brighter $1.25 \mathrm{mag}$ in $I_{A B}$ than 
Table B.1. Schechter parameters $\left(M^{*}, \alpha, \phi^{*}\right)$ and total luminosity density LD for the rest-frame FUV- and NUV-band galaxy luminosity function for the VVDS Deep+Ultra-Deep sample with the cosmology $\left(\Omega_{\mathrm{m}}, \Omega_{\Lambda}, h\right)=(0.3,0.7,0.7)$.

\begin{tabular}{|c|c|c|c|c|c|c|c|c|}
\hline \multirow[b]{2}{*}{ Redshift range $^{a}$} & \multicolumn{4}{|c|}{$\overline{\text { FUV }}$} & \multicolumn{4}{|c|}{ NUV } \\
\hline & $\begin{array}{c}M_{\mathrm{FUV}}^{*} \\
(\mathrm{AB} \text { mag) }\end{array}$ & $\alpha$ & $\begin{array}{c}\phi^{*} \\
\left(10^{-3} \mathrm{Mpc}^{-3}\right)\end{array}$ & $\begin{array}{c}\lg \left(\mathrm{LD}_{\mathrm{uc}}\right)^{b} \\
\mathrm{~W} / \mathrm{Hz} / \mathrm{Mpc}^{3}\end{array}$ & $\begin{array}{c}M_{\mathrm{NUV}}^{*} \\
(\mathrm{AB} \text { mag) }\end{array}$ & $\alpha$ & $\begin{array}{c}\phi^{*} \\
\left(10^{-3} \mathrm{Mpc}^{-3}\right)\end{array}$ & $\begin{array}{c}\lg \left(\mathrm{LD}_{\mathrm{uc}}\right)^{b} \\
\mathrm{~W} / \mathrm{Hz} / \mathrm{Mpc}^{3}\end{array}$ \\
\hline $\begin{array}{c}0.05<z \leq 0.2 \\
-\quad 0.2<z \leq 0.4 \\
\bullet \quad 0.4<z \leq 0.6 \\
-\quad 0.6<z \leq 0.8 \\
-\quad 0.8<z \leq 1.0 \\
-\quad 1.0<z \leq 1.2 \\
-\quad 1.2<z \leq 1.7 \\
1.7<z \leq 2.5 \\
2.5<z \leq 3.5 \\
3.5<z \leq 4.5\end{array}$ & $\begin{array}{c}-18.6_{-0.6}^{+0.4} \\
-18.3_{-0.2}^{+0.1} \\
-18.4_{-0.1}^{+0.1} \\
-18.3_{-0.1}^{+0.1} \\
-18.7_{-0.1}^{+0.1} \\
-19.0_{-0.2}^{+0.2} \\
-19.6_{-0.2}^{+0.2} \\
-20.7_{-0.7}^{+0.4} \\
-20.8_{-0.3}^{+0.3} \\
-\end{array}$ & $\begin{array}{c}-1.10_{-0.06}^{+0.06} \\
-1.17_{-0.05}^{+0.05} \\
-1.07_{-0.06}^{+0.07} \\
-0.90_{-0.08}^{+0.08} \\
-0.85_{-0.10}^{+0.10} \\
-0.91_{-0.16}^{+0.16} \\
-1.09_{-0.23}^{+0.23} \\
-1.65_{-0.53}^{+0.55} \\
-0.63_{-0.53}^{+0.57} \\
-\end{array}$ & $\begin{array}{c}5.36_{-1.46}^{+1.57} \\
6.91_{-0.05}^{+1.02} \\
6.60_{-0.96}^{+0.91} \\
9.53_{-0.99}^{+0.99} \\
9.01_{-0.96}^{+0.94} \\
7.43_{-1.15}^{+1.08} \\
4.10_{-0.87}^{+0.77} \\
2.23_{-1.56}^{+1.85} \\
1.72_{-0.19}^{+0.19} \\
-\end{array}$ & $\begin{array}{c}\text { Free Schech } \\
18.86_{-0.13}^{+0.25} \\
18.87_{-0.03}^{+0.03} \\
18.85_{-0.02}^{+0.02} \\
18.93_{-0.01}^{+0.02} \\
19.04_{-0.01}^{+0.02} \\
19.12_{-0.04}^{+0.04} \\
19.13_{-0.07}^{+0.12} \\
19.70_{-0.36}^{+3.25} \\
19.17_{-0.10}^{+0.25} \\
-\end{array}$ & $\begin{array}{c}\text { parameter } \\
-18.5_{-0.4}^{+0.3} \\
-18.3_{-0.1}^{+0.1} \\
-18.7_{-0.1}^{+0.1} \\
-18.8_{-0.1}^{+0.1} \\
-19.2_{-0.1}^{+0.1} \\
-19.6_{-0.1}^{+0.1} \\
-20.2_{-0.1}^{+0.1} \\
-20.7_{-0.4}^{+0.3} \\
-21.4_{-0.3}^{+0.3} \\
-\end{array}$ & $\begin{array}{c}-1.08_{-0.06}^{+0.06} \\
-1.02_{-0.04}^{+0.04} \\
-1.08_{-0.05}^{+0.05} \\
-0.95_{-0.08}^{+0.08} \\
-0.81_{-0.08}^{+0.09} \\
-0.88_{-0.12}^{+0.13} \\
-1.05_{-0.16}^{+0.17} \\
-1.16_{-0.45}^{+0.47} \\
-1.15_{-0.40}^{+0.42} \\
-\end{array}$ & $\begin{array}{c}6.35_{-1.47}^{+1.59} \\
10.16_{-1.01}^{+1.06} \\
6.82_{-0.79}^{+0.84} \\
9.27_{-1.01}^{+1.01} \\
9.48_{-0.88}^{+0.87} \\
6.57_{-0.89}^{+0.86} \\
3.49_{-0.58}^{+0.58} \\
3.73_{-1.29}^{+1.67} \\
1.32_{-0.47}^{+0.37} \\
-\end{array}$ & $\begin{array}{c}18.90_{-0.11}^{+0.17} \\
18.97_{-0.03}^{+0.03} \\
18.99_{-0.02}^{+0.02} \\
19.12_{-0.02}^{+0.02} \\
19.27_{-0.01}^{+0.01} \\
19.30_{-0.02}^{+0.02} \\
19.28_{-0.04}^{+0.06} \\
19.56_{-0.14}^{+0.52} \\
19.37_{-0.13}^{+0.38} \\
-\end{array}$ \\
\hline - $\quad 0.05<z \leq 0.2$ & \multicolumn{8}{|c|}{$\begin{array}{l}M^{*} \text { constrained }{ }^{c}, \alpha \text { and } \phi^{*} \text { free } \\
18.76_{-0.04}^{+0.04}\end{array}$} \\
\hline $\begin{array}{l}\bullet \quad 1.7<z \leq 2.5 \\
\cdot \quad 2.5<z \leq 3.5 \\
\cdot \quad 3.5<z \leq 4.5 \\
1.7<z \leq 2.5 \\
2.5<z \leq 3.5 \\
3.5<z \leq 4.5 \\
1.7<z \leq 2.5 \\
2.5<z \leq 3.5\end{array}$ & $\begin{array}{l}-20.4_{-0.1}^{+0.1} \\
-21.4_{-0.1}^{+0.1} \\
-22.2_{-0.2}^{+0.2} \\
-20.3_{-0.1}^{+0.1} \\
-21.1_{-0.1}^{+0.1} \\
-21.8_{-0.1}^{+0.1} \\
-20.8_{-0.2}^{+0.2} \\
-21.6_{-0.1}^{+0.1}\end{array}$ & $\begin{array}{l}-1.30 \\
-1.50 \\
-1.73 \\
-1.10 \\
-1.10 \\
-1.10 \\
-1.73 \\
-1.73 \\
\end{array}$ & $\begin{array}{l}3.37_{-0.24}^{+0.24} \\
0.86_{-0.05}^{+0.05} \\
0.11_{-0.01}^{+0.01} \\
3.94_{-0.28}^{+0.28} \\
1.27_{-0.07}^{+0.07} \\
0.22_{-0.02}^{+0.02} \\
1.95_{-0.14}^{+0.14} \\
0.60_{-0.03}^{+0.03} \\
\end{array}$ & $\begin{array}{c}\alpha \text { constrained }^{d} \\
19.46_{-0.03}^{+0.03} \\
19.40_{-0.02}^{+0.02} \\
19.10_{-0.05}^{+0.06} \\
19.41_{-0.02}^{+0.02} \\
19.24_{-0.02}^{+0.02} \\
18.73_{-0.03}^{+0.03} \\
19.79_{-0.04}^{+0.05} \\
19.62_{-0.03}^{+0.03} \\
\end{array}$ & $M^{*}$ and $\phi^{*} \mathrm{f}$ & & & \\
\hline
\end{tabular}

Notes. ${ }^{(a)}$ The entries labelled with a filled dot are the chosen Schechter parameters taken for the rest-frame FUV LF used throughout the paper, and reported in Table $1 .{ }^{(b)}$ The quoted errors are those induced by the STY LF fit; see Table 1 for errors including also other sources of uncertainty. ${ }^{(c)} M^{*}$ is set at $z \leq 0.2$ assuming the local rest-frame FUV value determined by Wyder et al. (2005). ${ }^{(d)} \alpha$ is set with different values at $z>1.7$. First, following an evolution with redshift as described in Sect. $3.2(\alpha=-1.3,-1.5,-1.73$ at $1.7<z \leq 2.5,2.5<z \leq 3.5,3.5<z \leq 4.5$, respectively); secondly, set as two likely extreme non-evolving values ( $\alpha=-1.1$ and $\alpha=-1.73$ ). We remind that $\alpha=-1.1$ corresponds to what we find at $1.2<z \leq 1.7$ (where we can still determine $\alpha$ with a reasonable error), and $\alpha=-1.73$ is the value found by Bouwens et al. (2007) at $z=3.8$. Contrarily to the rest-frame FUV LF, we were not interested in setting any Schechter parameters for the rest-frame NUV LF.

the zphot sample) does not significantly miss any faint galaxies, which strengthens the reliability of our flat faint-end slope. One small discrepancy occurs at $z \leq 0.2$, where the photometric $1 / V_{\max }$ points have an higher normalisation with a slightly steeper slope. It is due to the well known degeneracy in the computation of photometric redshifts, i.e., some objects end up with a wrong too low (zphot $\lesssim 0.3$ ) photometric redshift (see e.g. Ilbert et al. 2009). Furthermore, it confirms once more that our weighting scheme is adequate. This strengthens also the reliability of our low $\phi^{*}$ value at $3.5<z \leq 4.5$, which is neither caused by the cut at $I_{A B}=24.75$ nor by an incomplete weighting scheme. We refer the reader to Sect. 3.3.2 and to McCracken et al. (2003) for a discussion about photometric completeness with respect to $I_{A B}$-band magnitude and surface brightness.

\section{B.2. The rest-frame FUV LF of starburst galaxies}

We are interested to check whether the flatness of our slope at $z<1.7$ could be due to a very low density of intrinsically reddish galaxies, which could have hidden, in the total sample, very high densities of faint intrinsically blue galaxies. Indeed an I-band selection does not select only starbursting galaxies, in contrast with $U V$-band selected samples like GALEX (Arnouts et al. 2005).
We have classified our galaxies of the VVDS Deep+UltraDeep spectroscopic redshift sample according the VVDS scheme of four photometric types, as described in Zucca et al. (2006). Here, we consider the "Type 4" galaxies, corresponding to galaxies for which the best template fit over the $u^{*} g^{\prime} r^{\prime} i^{\prime} z^{\prime} J H K_{\mathrm{s}}$ broad bands is among the bluest galaxy templates, i.e., a starburst or irregular galaxy template. The Type 4 rest-frame FUV-band $1 / V_{\max }$ LF estimates are shown in Fig. B.2. At $z<1.7$, their normalisation is obviously lower with respect to the total sample, because the fraction of Type 4 galaxies in our sample goes from $\sim 40 \%$ at $z \sim 0.1$ up to $\sim 85 \%$ at $z \sim 4$ (when considering galaxies brighter than the bias limit). The faint-end LF slope for Type 4 galaxies is very similar to the one found for the total sample. Our flat slope is obviously not due to very low densities of faint intrinsically red galaxies, which could have overcompensate very high densities of faint intrinsically blue galaxies. Thus, it reinforces the flatness of our slope.

\section{B.3. The rest-frame NUV LF}

The last point to test is the fact that our rest-frame FUV magnitudes are based on template extrapolations at $z \leq 1$, which could have an impact on the faint-end LF slope. Our bluest photometric information is in the $u^{*}-381$ CFHTLS filter, and thus 


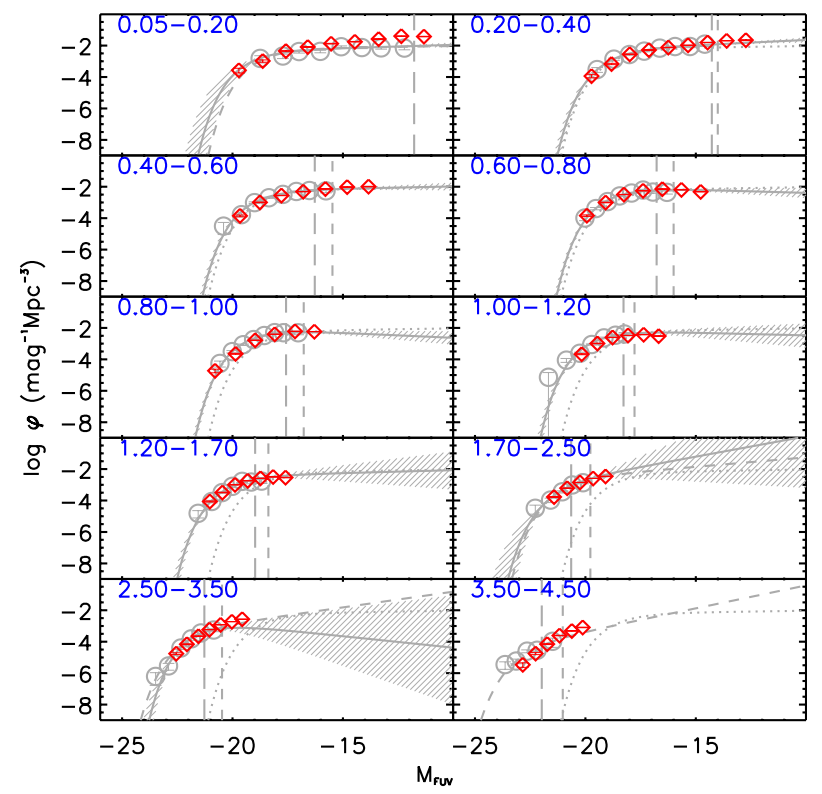

Fig. B.1. Rest-frame FUV $1 / V_{\max }$ LF estimates from $z=0.05$ to $z=$ 4.5 derived with the VVDS $z$ phot catalogue up to $I_{A B}=26$ (red open diamonds). They are plotted over a grey-scale version of Fig. 1, that is the rest-frame FUV LF of the VVDS Deep+Ultra-Deep spectroscopic sample up to $I_{A B}=24.75$.

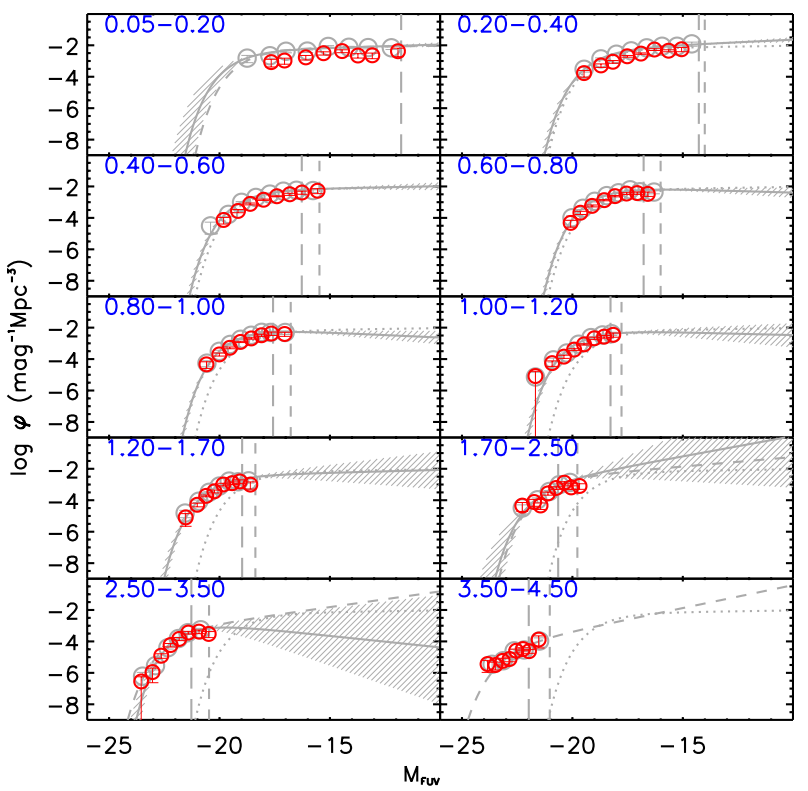

Fig. B.2. Rest-frame FUV $1 / V_{\max }$ LF estimates from $z=0.05$ to $z=4.5$ for our intrinsically bluest galaxies (red open circles, VVDS Type 4, see text) in the VVDS Deep+Ultra-Deep spectroscopic sample. They are plotted over a grey-scale version of Fig. 1, that is the rest-frame FUV LF of the total VVDS Deep+Ultra-Deep spectroscopic sample.

the rest-frame FUV emission is directly observed from $z \geq 1$. Nevertheless, the rest-frame NUV emission is directly observed at $z \geq 0.4$. Thus we report our results from the rest-frame NUV-band LF of the Deep+Ultra-Deep spectroscopic sample. We list the Schechter parameters derived with the STY method, in Table B.1, and plot the LFs in Fig. B.3. We note that we were not interested in trying to fix $M *$ at low $z$, nor to fix $\alpha$ at very high $z$. These estimates enable us to measure the faint-end slope at $0.4 \lesssim z \lesssim 1$, that is where the FUV intrinsic luminosity is extrapolated but not the NUV intrinsic luminosity. Again, we do

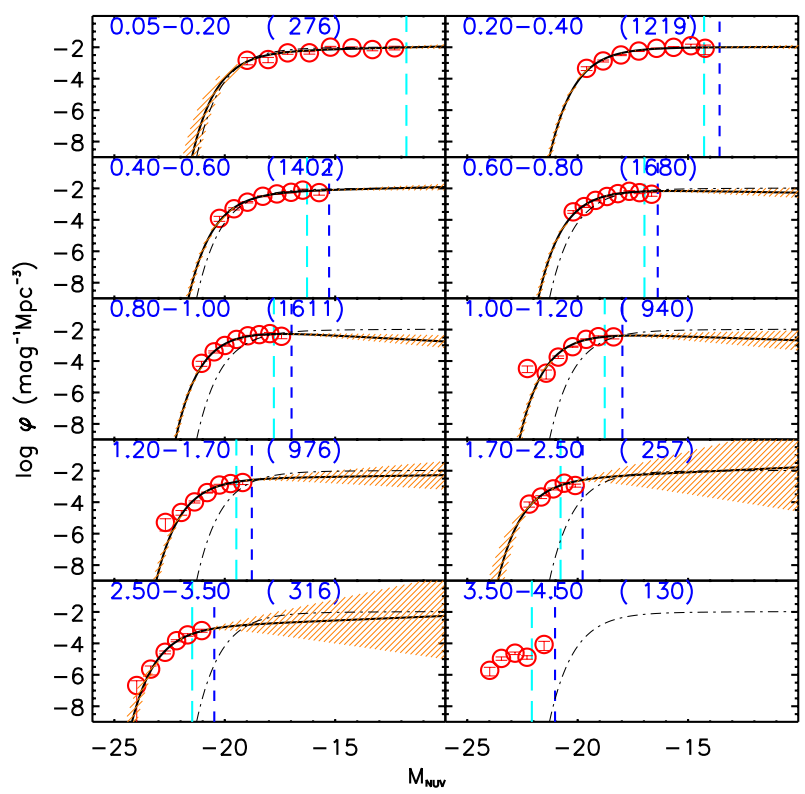

Fig. B.3. Rest-frame NUV-band LFs from $z=0.05$ to $z=4.5$ of the VVDS Deep+Ultra-Deep sample, in 10 redshift bins as indicated in each panel. Red circles represent the $1 / V_{\max }$ data points up to the LF bias limit and the corresponding number of galaxies are given in parenthesis. The vertical cyan long-dashed and blue short-dashed lines correspond to the LF bias limits for the Deep and Ultra-Deep surveys, respectively. The black solid curve and the associated shaded area is the STY LF estimate assuming free Schechter parameters and its associated error. The dot-dashed curve is the same in each panel, and it corresponds to the solid curve in the $0.2<z<0.4$ redshift bin, as reference. All Schechter parameters are given in Table B.1. For the NUV-band, we were not interested in fixing either $M^{*}$ nor $\alpha$. We note that in the last redshift bin the STY fit is unconstrained, so there is no solid curve overplotted.

not find a steeper faint-end slope in the rest-frame NUV-band LFs. As our aim is to derive the SFRD from the rest-frame ultraviolet continuum spanning from FUV to the NUV using the relation of Kennicutt (1998), we can affirm that the extrapolation of the FUV-band luminosities does not change our SFRD results since the faint-end slope stays flat.

\section{Appendix C: FUV number counts and luminosity functions at $z \sim 3$}

At $2.7 \leq z \leq 3.4$, Le Fèvre et al. (2005a) and Le Fèvre et al. (in prep.) show that the VVDS presents number counts per $0.5 \mathrm{mag}$ interval of $I_{A B}$ apparent magnitude per unit surface area (i.e., $\left.\mathrm{d} N / 0.5 \mathrm{mag} / \mathrm{arcmin}^{2}\right)$ at least 2 times larger than those quoted by Steidel et al. (1999) within the same redshift range. This is particularly evident for bright galaxies $\left(I_{A B} \lesssim 23.5\right)$. In contrast, the VVDS rest-frame FUV LF is only $\sim 50 \%$ higher than the one found by Steidel et al. (1999), as illustrated in the left panel of our Fig. C.1, and this holds only for $M_{\mathrm{FUV}}<-22.5$. Here, we demonstrate that these two results are not in conflict. We remark that throughout the paper we compute our VVDS FUV absolute magnitudes using a filter centred at $1500 \AA$, while Steidel et al. (1999) use a FUV-band filter centred at $1700 \AA$. To compare our results with their work, in this section we compute our VVDS FUV absolute magnitudes using the same filter as Steidel et al. (1999), even though our VVDS FUV-150 and VVDS-1700 LFs are very similar. 
Table B.2. Non exhaustive list of the faint-end slope values, $\alpha$, of the rest-frame FUV-band luminosity functions at $0<z<6$, as found in the literature.

\begin{tabular}{|c|c|c|c|c|c|c|c|}
\hline Reference & $z^{a}$ & 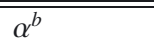 & $\overline{\lambda \lambda}$ & Reference & 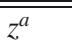 & 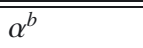 & $\overline{\lambda \lambda}$ \\
\hline Wyder et al. (2005) & 0.055 & $-1.22_{-0.07}^{+0.07}$ & 1500 & Wilson et al. (2002) ${ }^{d}$ & 1.35 & -1.5 & 2500 \\
\hline This work VVDS Deep ${ }^{c}$ & 0.125 & $-1.05_{-0.04}^{+0.04}$ & 1500 & Gabasch et al. (2004) & 1.36 & -1.07 & 1500 \\
\hline Tresse et al. (2007) VVDS Deep ${ }^{c}$ & 0.14 & $-1.13_{-0.11}^{+0.10}$ & 1500 & This work VVDS Deep+Ultra-Deep & 1.45 & $-1.09_{-0.23}^{+0.23}$ & 1500 \\
\hline Sullivan et al. $(2000)^{d}$ & 0.15 & -1.51 & 2000 & Oesch et al. (2010) & 1.5 & $-1.86_{-0.48}^{+0.48}$ & 1500 \\
\hline Treyer et al. (1998) ${ }^{d}$ & 0.15 & -1.62 & 2000 & Tresse et al. (2007) & 1.55 & -1.6 & 1500 \\
\hline Arnouts et al. (2005) GALEX-VVDS & 0.3 & $-1.19_{-0.15}^{+0.15}$ & 1500 & Sawicki \& Thompson (2006) & 1.7 & $-0.81_{-0.15}^{+0.21}$ & 1700 \\
\hline This work VVDS Deep+Ultra-Deep & 0.3 & $-1.17_{-0.05}^{+0.05}$ & 1500 & Connolly et al. (1997) $)^{d}$ & 1.75 & $-1.3^{-0.13}$ & 2800 \\
\hline Tresse et al. (2007) & 0.3 & $-1.6^{-0.05}$ & 1500 & Oesch et al. (2010) & 1.75 & $-1.72_{-0.15}^{+0.15}$ & 1500 \\
\hline Wilson et al. $(2002)^{d}$ & 0.35 & -1 & 2500 & Gabasch et al. (2004) & 1.88 & -1.07 & 1500 \\
\hline Wilson et al. $(2002)^{d}$ & 0.35 & -1.5 & 2500 & Oesch et al. (2010) & 1.9 & $-1.59_{-0.52}^{+0.52}$ & 1500 \\
\hline Arnouts et al. (2005) GALEX-VVDS & 0.5 & $-1.55_{-0.21}^{+0.21}$ & 1500 & Arnouts et al. (2005) & 2 & $-1.49_{-0.24}^{+0.24}$ & 1500 \\
\hline This work VVDS Deep+Ultra-Deep & 0.5 & $-1.07_{-0.06}^{+0.07}$ & 1500 & This work VVDS Deep+Ultra-Deep & 2.1 & -1.3 & 1500 \\
\hline Tresse et al. (2007) & 0.51 & $-1.6^{-0.00}$ & 1500 & Sawicki \& Thompson (2006) & 2.2 & $-1.20_{-0.22}^{+0.24}$ & 1700 \\
\hline Gabasch et al. (2004) & 0.63 & -1.07 & 1500 & Reddy \& Steidel (2009) & 2.3 & $-1.73_{-0.07}^{+0.07}$ & 1700 \\
\hline Tresse et al. (2007) & 0.69 & -1.6 & 1500 & Oesch et al. (2010) & 2.5 & $-1.76_{-0.14}^{+0.14}$ & 1500 \\
\hline Arnouts et al. (2005) GALEX-VVDS & 0.7 & $-1.60_{-0.26}^{+0.26}$ & 1500 & Gabasch et al. (2004) & 2.53 & -1.07 & 1500 \\
\hline Cowie et al. (1999) ${ }^{d}$ & 0.7 & $-1{ }^{-0.20}$ & 2000 & Arnouts et al. (2005) & 2.9 & $-1.47_{-0.21}^{+0.21}$ & 1500 \\
\hline Cowie et al. (1999) ${ }^{d}$ & 0.7 & -1.5 & 2000 & This work VVDS Deep+ultra-Deep & 3 & $-1.5^{-0.21}$ & 1500 \\
\hline This work VVDS Deep+Ultra-Deep & 0.7 & $-0.90_{-0.08}^{+0.08}$ & 1500 & Sawicki \& Thompson (2006) & 3 & $-1.43_{-0.09}^{+0.17}$ & 1700 \\
\hline Connolly et al. (1997) $)^{d}$ & 0.75 & -1.3 & 2800 & Steidel et al. (1999) & 3.04 & -1.6 & 1700 \\
\hline Oesch et al. (2010) & 0.75 & $-1.54_{-026}^{+0.26}$ & 1500 & Tresse et al. (2007) VVDS Deep & 3.04 & -1.6 & 1500 \\
\hline Wilson et al. (2002) ${ }^{d}$ & 0.8 & -1 & 2500 & Reddy \& Steidel (2009) & 3.05 & $-1.73_{-0.13}^{+0.13}$ & 1700 \\
\hline Wilson et al. (2002) ${ }^{d}$ & 0.8 & -1.5 & 2500 & Gabasch et al. (2004) & 3.46 & $-1.07^{-0.13}$ & 1500 \\
\hline This work VVDS Deep+Ultra-Deep & 0.9 & $-0.85_{-0.10}^{+0.10}$ & 1500 & Paltani et al. (2007) VVDS Deep & 3.5 & -1.4 & 1700 \\
\hline Tresse et al. (2007) VVDS Deep & 0.9 & -1.6 & 1500 & Tresse et al. (2007) VVDS Deep & 3.6 & -1.6 & 1500 \\
\hline Gabasch et al. (2004) & 0.96 & -1.07 & 1500 & Bouwens et al. (2007) & 3.8 & $-1.73_{-0.05}^{+0.05}$ & 1600 \\
\hline Arnouts et al. (2005) GALEX-VVDS & 1 & $-1.63_{-0.45}^{+0.45}$ & 1500 & This work VVDS Deep+Ultra-Deep & 4 & -1.73 & 1500 \\
\hline Tresse et al. (2007) VVDS Deep & 1.09 & -1.6 & 1500 & Sawicki \& Thompson (2006) & 4 & $-1.26_{-0.36}^{+0.40}$ & 1700 \\
\hline This work VVDS Deep+Ultra-Deep & 1.1 & $-0.91_{-0.16}^{+0.16}$ & 1500 & Steidel et al. (1999) & 4.13 & -1.6 & 1700 \\
\hline Connolly et al. $(1997)^{d}$ & 1.25 & -1.3 & 2800 & Tresse et al. (2007) VVDS Deep & 4.26 & -1.6 & 1500 \\
\hline Cowie et al. (1999) ${ }^{d}$ & 1.25 & -1 & 2000 & Gabasch et al. (2004) & 4.51 & -1.07 & 1500 \\
\hline Cowie et al. (1999) ${ }^{d}$ & 1.25 & -1.5 & 2000 & Bouwens et al. (2007) & 5 & $-1.66_{-0.09}^{+0.09}$ & 1600 \\
\hline Oesch et al. (2010) & 1.25 & $-1.76_{-0.23}^{+0.23}$ & 1500 & Iwata et al. (2007) & 5 & $-1.48_{-0.32}^{+0.38}$ & 1700 \\
\hline Tresse et al. (2007) VVDS Deep & 1.29 & -1.6 & 1500 & Bouwens et al. (2007) & 5.9 & $-1.74_{-0.16}^{+0.16}$ & 1350 \\
\hline Wilson et al. $(2002)^{d}$ & 1.35 & -1 & 2500 & & & & \\
\hline
\end{tabular}

Notes. Values are sorted according to the redshift. The effective wavelength of the rest-frame band at which the LF was determined is also given. (a) Mean or median redshift as quoted in each work, or, if not specified, centre of the studied redshift interval. ${ }^{(b)}$ Faint-end slope of the UV LF, with error bars when it was estimated, with no error bars when it was fixed. ${ }^{(c)}$ Faint-end slope of the nearby FUV LF estimated after setting $M_{\mathrm{FUV}}^{*}$. (d) Value retrieved from the compilation of Hopkins (2004) .

As Steidel et al. (1999) begin their analysis from galaxy number counts as a function of $\mathcal{R}$ apparent magnitude, we overplot the same for the VVDS Deep and Ultra-Deep surveys in the right panel of Fig. C.1. We remark that in this figure we plot the number of galaxies per unit magnitude and per unit volume (i.e., $\mathrm{d} N / \mathrm{mag} / \mathrm{Mpc}^{3}$ ), while Steidel et al. (1999) cite in their Table 3 the surface density number counts for $0.5 \mathrm{mag}$ intervals $\left(\mathrm{d} N / 0.5 \mathrm{mag} / \mathrm{arcmin}^{2}\right)$. To convert their values in $\mathrm{d} N / \mathrm{mag} / \mathrm{Mpc}^{3}$ units, we multiplied them $\times 2$ to obtain the correct magnitude interval, and we divided for the effective volume of each $\operatorname{arcmin}^{2}$, quoted in their Table 3 (with the same cosmology that we adopt, $\left.\Omega_{m}=0.3, \Omega_{\Lambda}=0.7\right)$. Our number counts are weighted as described in Appendices A.1 and A.2, and they are realized as a function of $m_{\mathrm{ST}}=(r+i) / 2$ apparent magnitude, i.e., the mean value of the $r$ - and $i$-CFHTLS broadband filters, because it mimics the $\mathcal{R}$-broadband filter used by Steidel et al. (1999). We observe that the VVDS counts are clearly higher than those from Steidel et al. (1999), at least at the brightest magnitudes.

Next, from their number counts, Steidel et al. (1999) derive their rest-frame FUV LF (shown in the left panel of Fig. C.1), translating apparent magnitudes into absolute magnitudes with the following rigid shift:

$M_{\mathrm{FUV}}=m_{\mathcal{R}}-D M\left(z_{\mathrm{med}}\right)+2.5 \log \left(z_{\mathrm{med}}+1\right)$,

where $\operatorname{DM}\left(z_{\text {med }}\right)$ is the distance modulus defined as $5 \times$ $\log \left(D_{L}\left(z_{\mathrm{med}}\right) / 10 \mathrm{pc}\right)$, with $D_{L}\left(z_{\mathrm{med}}\right)$ the luminosity distance at $z_{\text {med }}$, and $z_{\text {med }}$ the median redshift of the studied sample. For their sample at $2.7 \leq z \leq 3.4\left(z_{\text {med }}=3.04\right)$, it gives:

$M_{\mathrm{FUV}}=m_{\mathcal{R}}-45.55$. 

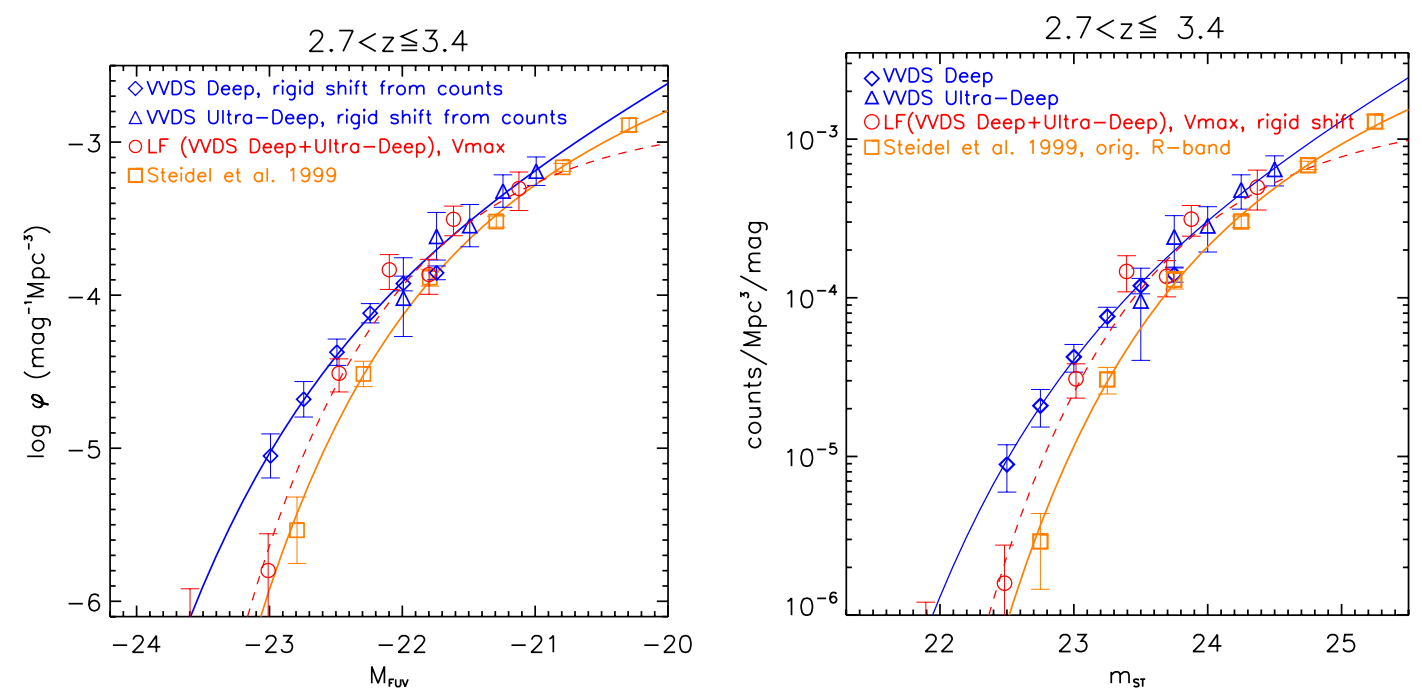

Fig. C.1. Left panel. Comoving luminosity densities as a function of FUV (1700 $\AA$ ) absolute luminosities at $2.7 \leq z \leq 3.4: 1 / V_{\max }$ estimates of our VVDS Deep+Ultra-Deep sample fitted with a Schechter function (red circles and dashed curve, respectively); our $m_{\mathrm{ST}}=(r+i) / 2$ number counts per volume density for the Deep and Ultra-Deep surveys converted into FUV absolute magnitudes with a rigid shift (see text), and fitted with a Schechter function (blue diamonds and triangles, and solid line, respectively); the $m_{\mathrm{ST}}=\mathcal{R}$ number counts per volume density of Steidel et al. (1999) converted into FUV absolute magnitudes with their rigid shift (see text), and fitted with a Schechter function by them (orange squares and solid line, respectively). Right panel. Number counts per unit volume of galaxies as a function of $m_{\mathrm{ST}}$ apparent magnitudes at $2.7 \leq z \leq 3.4$ : our number counts from the VVDS Deep and Ultra-Deep surveys (blue diamonds and triangles, respectively); our VVDS number counts derived from our VVDS $1 / V_{\max }$ LF (see right panel) shifted rigidly in magnitude (red circles, see text); number counts from Steidel et al. (1999) (orange squares; see text for conversion details). The three curves correspond to the same curves in the left panel, shifted rigidly (see text). Note that the CFHTLS $(r+i) / 2$ photometric system mimics the $\mathcal{R}$ one used by Steidel et al. (1999).

For the VVDS Deep+Ultra-Deep sample, at $2.7 \leq z \leq 3.4$ we have $z_{\text {med }}=2.92$. With the same method, starting from our observed number counts (see right panel of Fig. C.1), we obtain the rest-frame FUV LF data points, as illustrated in the left panel of Fig. C.1, and we fitted them with a Schechter function. The result is that our $\mathrm{d} N$-derived LF is higher at the bright end than our direct $1 / V_{\max }$ LF data points, as derived in Sect. 3.2, and here fitted also with a Schechter function. We conclude that our $N$-derived LF presents a different shape than our $1 / V_{\max }$-derived LF.

Below we verify that, as expected, it is not correct to use a simple, rigid shift to transform our apparent magnitudes to absolute ones, as done with Eq. (C.1), to obtain correct $\mathrm{d} N$-derived LFs. The main reasons are the following.

1) The $n(z)$ shape. In the range $2.7 \leq z \leq 3.4$, the redshift distribution $n(z)$ of an $I$-band selected survey as observed by the VVDS decreases as a function of $z$ as shown in Le Fèvre et al. (in prep.), in particular in a faster way for brighter galaxies. In contrast, due to the colour selection function used to find Lyman-break galaxies, the $n(z)$ in Steidel et al. (1999) peaks around its median value within the same redshift range. The shapes of the different $n(z)$ are shown in the inset of Fig. C.2. If the $n(z)$ is skewed, it is crucial to use the correct redshift distribution in Eq. (C.1), and not simply the median redshift value.

2) The $K$ - and colour corrections. Steidel et al. (1999) restrict the $(K+$ colour $)$ term to $2.5 \log (1+z)$, as in Eq. (C. 1$)$, because they work with the observed $\mathcal{R}$-band, corresponding to the FUV light emitted at $z \sim 3$. Nevertheless, this is correct for $z \sim 3.0$ galaxies only.

We made a simple simulation to illustrate these two aspects. We started with a set of $3 \times 10^{6}$ galaxies distributed in FUV absolute magnitude like the Schechter fit of our $1 / V_{\max }$-derived FUV LF. We want to know whether, with a selection function as the VVDS, we recover our observed galaxy number counts as a function of $m_{\mathrm{ST}}$ apparent magnitude at $2.7 \leq z \leq 3.4$. Using Eq. (C.1), we operate a rigid shift to transform our FUV absolute magnitudes into $m_{\mathrm{ST}}$ apparent magnitudes, and to obtain a LF-derived number counts as illustrated in the right panel of Fig. C.1. We call this LF-derived distribution $n\left(m_{\mathrm{ST}}, L F\right)$. We see that these LF-derived number counts are not in agreement with our observed number counts which are at least twice higher at $m_{\mathrm{ST}}<23$. We illustrate in Fig. C.2 the ratio of our observed number counts to our $n\left(m_{\mathrm{ST}}, L F\right)$ as a function of $m_{\mathrm{ST}}$.

We will now test the $n(z)$ and the $(K+$ colour $)$ corrections, respectively. We fit separately the $n(z)$ of VVDS galaxies with $m_{\mathrm{ST}} \leq 23$ and with $m_{\mathrm{ST}}>23$, to account for the steeper $n(z)$ decrease for bright magnitudes (see inset in Fig. C.2). Given the monotonic (even if scattered) relation between observed and absolute magnitudes, $m_{\mathrm{ST}} \sim 23$ corresponds to $M_{\mathrm{FUV}} \sim-21.1$. Thus, for each previously simulated galaxy, we assign a redshift in respecting the different $n(z)$ distributions for galaxies brighter or fainter than $M_{\mathrm{FUV}}=-21.1$, that is we do not anymore assume a single median redshift. We now compute the observed $m_{\mathrm{ST}}$ magnitudes of our simulated sample using Eq. (C.1), where we substitute $z_{\text {med }}$ with the particular redshift that has been assigned to each galaxy. We obtain a $m_{\mathrm{ST}}$ distribution that we call $n\left(m_{\mathrm{ST}}, z\right)$. The ratio of our observed number counts to the $n\left(m_{\mathrm{ST}}, z\right)$ is shown in Fig. C.2. The $n\left(m_{\mathrm{ST}}, z\right)$ distribution is now closer to our observed number counts than the $n\left(m_{\mathrm{ST}}, L F\right)$ distribution.

As a further step, we account for the $(K+$ colour $)$ terms for each previously simulated galaxy, that is:

$M_{\mathrm{FUV}}=m_{\mathrm{ST}}-D M(z)-K_{\mathrm{ST}}(z)-\left(m_{\mathrm{ST}}-m_{\mathrm{FUV}}\right)^{(S E D, z=0)}$,

where $K_{\mathrm{ST}}(z)$ is the $K$-correction at $z$ and $\left(m_{\mathrm{ST}}-m_{\mathrm{FUV}}\right)$ is the colour term at $z=0$, both derived from the best SED fitting template. We have modelled the best fitting template distribution in the VVDS sample, which gives us the VVDS distributions of 
O. Cucciati et al.: The CSFRD since 12 Gyr traced by the VVDS

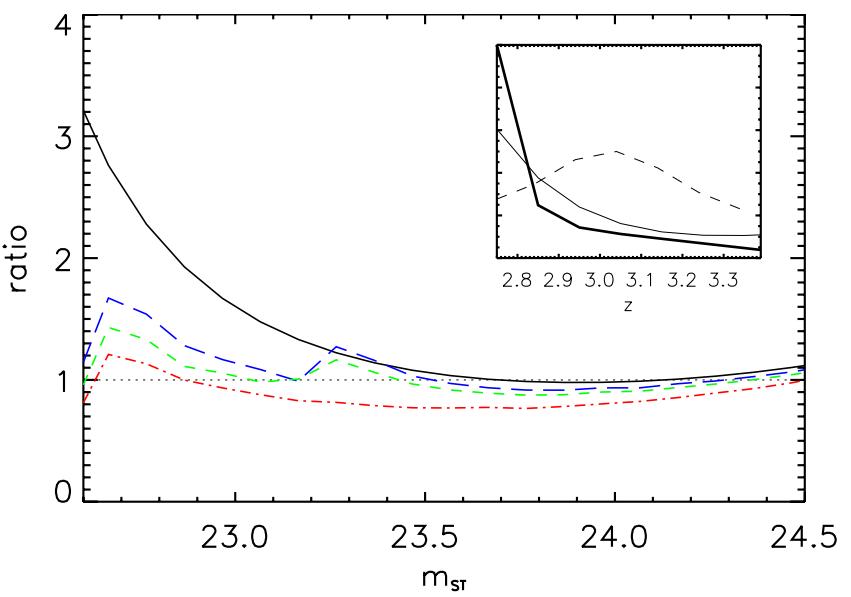

Fig. C.2. As a function of $m_{\mathrm{ST}}$, ratio of the directly observed VVDS FUV number counts at $2.7 \leq z \leq 3.4$ to the number counts derived in various ways from the FUV luminosity function in the same $z$ range. The black dotted line (ratio $=1$ ) is the ideal case where both counts are identical. The black solid line represents the ratio of the observed VVDS to the VVDS LF derived counts using Eq. (C.1), $n\left(m_{\mathrm{ST}}, L F\right)$ (i.e. blue solid line to the red dashed line of Fig. C.1, right panel). The other lines represent the ratio of the observed VVDS number counts (the above mentioned blue line) to the following various simulated distributions: $n\left(m_{\mathrm{ST}}, z\right)$ (blue long-dash curve), $n\left(m_{\mathrm{ST}}, z, K, \mathrm{col}\right)$ (green shortdash curve) and $n\left(m_{\mathrm{ST}}, \Delta M\right)$ (red dot-dash curve). See text for details about these simulated distributions. In the inset, we plot the global shape of the redshift distributions in Steidel et al. (1999) (dashed line) and in our work, separately for faint and bright galaxies (thin and thick solid lines, respectively). The normalisation on the $y$-axis is consistent between the two solid lines, while the normalisation of the dashed line has been arbitrarily chosen to better illustrate the different shapes.
$K_{\mathrm{ST}}(z)$ and $\left(m_{\mathrm{ST}}-m_{\mathrm{FUV}}\right)^{(S E D, z=0)}$ terms. Also in this case we assumed the two $n(z)$ distributions (for faint and bright galaxies) described above. Using the specific distribution of all the terms in Eq. (C.3), we computed a new set of $m_{\mathrm{ST}}$ starting from our sample of absolute magnitudes, and obtained their distribution $\left(n\left(m_{\mathrm{ST}}, z, K, \mathrm{col}\right)\right)$. We show in Fig. C. 2 the ratio of our observed number counts to the $n\left(m_{\mathrm{ST}}, z, K, \mathrm{col}\right)$ distribution. We observe that the modelisation of the $K+$ colour term further improves the match with the observed number counts. Still, the ratio is not equal to unity, which demonstrates that the reality is more complex than a simple simulated recipe.

To mimic the VVDS observed data, but still with a very simple test, we have fitted with a Gaussian function the distribution of $\Delta M=M_{\mathrm{FUV}}-m_{\mathrm{ST}}$ in the VVDS sample. Subtracting from each simulated $M_{\text {FUV }}$ a value of $\Delta M$ extracted randomly from the distribution, we obtained a new $m_{\mathrm{ST}}$ distribution, that we called $n\left(m_{\mathrm{ST}}, \Delta M\right)$. It is clear that this last distribution (see Fig. C.2) mimics better than the others the real observed number counts distribution at bright magnitudes, but then the match for fainter galaxies is slightly worse.

In summary, this simple exercise confirms that it is very dangerous to transform number counts within a given redshift range to a luminosity function in the case of a skewed $n(z)$ within the redshift interval considered, and/or in the case of dissimilar $n(z)$ for different galaxy populations. The fact that the $n(z)$ of colourselected samples is very different from the $n(z)$ of magnitude limited samples, implies that number counts when using colourselected samples are not representative of the number counts for the complete galaxy population. We refer the reader to Le Fèvre et al. (2005a) and Le Fèvre et al. (in prep.) for more details on number counts and sample selections. 